\title{
Postnatal lymph node expansion of stromal progenitors towards reticular and CD34+ stromal cell subsets is determined by distinct transcriptional programs
}

\section{Joern Pezoldt}

Suchergebnisse Webergebnis mit Sitelinks École polytechnique fédérale de Lausanne https://orcid.org/0000-0001-7143-5691

\section{Carolin Wiechrs}

Helmholtz Centre for Infection Research

Maria Litovchenko

École Polytechnique Fédérale de Lausanne (EPFL)

\section{Marjan Biočanin}

École Polytechnique Fédérale de Lausanne

\section{Mangge Zou}

Helmholtz Centre for Infection Research

\section{Katarzyna Sitnik}

Helmholtz Centre for Infection Research

\section{Michael Beckstette}

Helmholtz Centre for Infection Research

\section{Wanze Chen}

École Polytechnique Fédérale de Lausanne

\section{Vincent Gardeux}

Institute of Bioengineering, School of Life Sciences, École Polytechnique Fédérale de Lausanne (EPFL),

Lausanne https://orcid.org/0000-0001-8954-2161

\section{Stefan Floess}

Helmholtz Centre for Infection Research

\section{Maria Ebel}

Helmholtz Centre for Infection Research

\section{Julie Russeil}

École Polytechnique Fédérale de Lausanne https://orcid.org/0000-0002-0880-9483

\section{Panagiota Arampatzi}

University of Würzburg https://orcid.org/0000-0001-7925-8984

\section{Ehsan Vafardanejad}

Helmholtz Institute for RNA-based Infection Research 
Antoine-Emmanuel Saliba

Helmholtz Institute for RNA-based Infection Research https://orcid.org/0000-0001-8539-2784

\section{Bart Deplancke}

École Polytechnique Fédérale de Lausanne (EPFL) https://orcid.org/0000-0001-9935-843X Jochen Huehn ( $\nabla$ jochen.huehn@helmholtz-hzi.de )

Helmholtz Centre for Infection Research https://orcid.org/0000-0001-8071-1379

\section{Article}

Keywords: distinct transcriptional programs, postnatal lymph node expansion, stromal progenitors, CD34+ stromal cell

Posted Date: June 22nd, 2021

DOI: https://doi.org/10.21203/rs.3.rs-596484/v1

License: (c) (i) This work is licensed under a Creative Commons Attribution 4.0 International License. Read Full License

Additional Declarations: There is NO Competing Interest.

Version of Record: A version of this preprint was published at Nature Communications on November 24th, 2022. See the published version at https://doi.org/10.1038/s41467-022-34868-4. 
Postnatal lymph node expansion of stromal progenitors towards reticular and CD34 ${ }^{+}$ stromal cell subsets is determined by distinct transcriptional programs

Joern Pezoldt $^{1,2}$, Carolin Wiechers ${ }^{1}$, Maria Litovchenko ${ }^{2}$, Marjan Biocanin ${ }^{2}$, Mangge Zou ${ }^{1}$, Katarzyna Sitnik ${ }^{3}$, Michael Beckstette ${ }^{1,4}$, Wanze Chen $^{2}$, Vincent Gardeux ${ }^{2}$, Stefan Floess ${ }^{1}$, Maria Ebel $^{1}$, Julie Russeil ${ }^{2}$, Panagiota Arampatzi ${ }^{5}$, Ehsan Vafardanejad ${ }^{6}$, Antoine-Emmanuel Saliba $^{6}$, Bart Deplancke ${ }^{2, \#}$, Jochen Huehn ${ }^{1,7^{*}, \#}$

${ }^{1}$ Department Experimental Immunology, Helmholtz Centre for Infection Research, Braunschweig, Germany

${ }^{2}$ Laboratory of Systems Biology and Genetics, École Polytechnique Fédérale de Lausanne, Lausanne, Switzerland

${ }^{3}$ Department of Vaccinology, Helmholtz Centre for Infection Research, 38124 Braunschweig, Germany

${ }^{4}$ Department of Computational Biology for Individualised Medicine, Centre for Individualised Infection Medicine, Helmholtz Centre for Infection Research and Hannover Medical School, Hannover, Germany

${ }^{5}$ Core Unit Systems Medicine, University of Wuerzburg, 97080 Wuerzburg, Germany

${ }^{6}$ Helmholtz Institute for RNA-based Infection Research, 97080 Wuerzburg, Germany

${ }^{7}$ Cluster of Excellence RESIST (EXC 2155), Hannover Medical School, Hannover, Germany

\#Equally contributing senior authors

*Corresponding author:

Jochen Huehn (Jochen.Huehn@ @elmholtz-hzi.de) 


\begin{abstract}
Gut-draining mesenteric lymph nodes $(\mathrm{mLN})$ provide the framework and microenvironment to shape intestinal adaptive immune responses. We previously delineated transcriptional signatures in LN stromal cells (SC), pointing to tissue-specific variability in composition and immuno-modulatory function of SCs.

Here, we dissect the tissue-specific epigenomic DNA accessibility and CpG methylation landscape of LN non-endothelial SCs and identify a microbiota-independent core epigenomic signature of LN SCs. By combined analysis of transcription factor (TF) binding sites together with the gene expression profiles of non-endothelial SCs, we delineated TFs poising skindraining peripheral $\mathrm{LN}(\mathrm{pLN}) \mathrm{SCs}$ for pro-inflammatory responses. Furthermore, using scRNA-seq, we dissected the developmental trajectory of mLN SCs derived from postnatal to aged mice, identifying two distinct putative progenitors, namely $\mathrm{CD} 34^{+} \mathrm{SC}$ and fibroblastic reticular stromal cell (FRC) progenitors, which both feed the rapid postnatal LN expansion. Finally, we identified $\operatorname{Irf} 3$ as a key differentiation TF inferred from the epigenomic signature of mLN SCs that is dynamically expressed along the differentiation trajectories of FRCs, and validated $\operatorname{Irf} 3$ as a regulator of $\mathrm{Cxcl}^{+}$FRC differentiation.

Together, our data constitute a comprehensive transcriptional and epigenomic map of $\mathrm{mLN}$ development and dissect location-specific, microbiota-independent properties of mLN nonendothelial SCs. As such, our findings represent a valuable resource to identify core transcriptional regulators that impinge on the developing mLN early in life, thereby shaping long-lasting intestinal adaptive immune responses.
\end{abstract}




\section{Introduction}

The mammalian immune system is tasked to detect pathogenic incursions and maintain balanced immune responses. Failure to achieve equilibrium can result in the development of local and systemic overreactions to self and foreign antigens or higher susceptibility to infections. As lymph nodes (LNs) are the initial hub translating early innate responses into lasting adaptive antigen-specific immunity, their tissue-specific modulation of developing immune responses is of essence to calibrate immune responses throughout life. Nonetheless, it is astounding that tissue-specific immune responses evolve in LNs given that they derive from seemingly identical postnatal LN anlagen that are positioned throughout the body.

LNs start to develop prenatally as early as embryonic day (E)13 in mice, in a process tightly regulated by mesenchymal lymphoid tissue organizer (LTo) and hematopoietic lymphoid tissue inducer (LTi) cells, yielding primordial LN anlagen. ${ }^{1,2}$ Initial recruitment and retention of LTis is mediated by endothelial LTos (eLTo). ${ }^{3}$ The same eLTos subsequently activate $\mathrm{Cxcl} 13^{+}$ mesenchymal LTos (mLTo), further recruiting additional LTis in a Cxcl13 ${ }^{+}$-driven manner..$^{2-4}$ With the LN anlagen established by E17, containing a dense network of LTis and LTos, ${ }^{5}$ endothelial and mesenchymal cells further proliferate. Already at E18, lymphatic endothelial cells (LEC) have sufficiently expanded to envelope the core parenchyma of the LN, establishing the border to the surrounding tissue beneath the forming LN capsule. ${ }^{6}$ After birth, the influx of $\mathrm{T}$ cells and $\mathrm{B}$ cells drastically increases, requiring and driving the rapid expansion of the LN stromal cell (LNSC) compartment including non-endothelial SCs. The LN parenchyma becomes increasingly segregated as the B cell and T cell zones are established. ${ }^{7}$ While the cortex situated underneath the subcapsular sinus of the LN contains the B cell follicles, T cells and dendritic cells (DCs) interact in the paracortex beneath. ${ }^{8}$ Aside blood endothelial cells (BEC) and LECs, a third major SC population, the reticular fibroblastic stromal cells (FRCs) populate the adult LN. The FRC pool consist of several heterogeneous subsets, including follicular dendritic cells (FDCs), which infrastructurally organize B cell follicles, marginal reticular cells (MRC) below the subcapsular sinus, T cell zone reticular cells (TRC) and medullary FRCs located in the paracortex and the medulla, respectively. ${ }^{4}$ These FRCs are thought to develop from mLTos transitioning through a myofibroblastic precursor stage. ${ }^{9}$ Recent scRNA-seq profiling of LNSCs revealed that the podoplanin (Pdpn, gp38)-expressing SC compartment encompasses a distinct and heterogeneous population of non-endothelial $\mathrm{CD} 34^{+} \mathrm{SCs}$, which are located at the LN capsule or the adventitia of large vessels. ${ }^{10-12}$ While several lineage-tracing models have delineated the prenatal origin of non-endothelial LNSCs, ${ }^{13-}$ 15 the postnatal differentiation process of FRCs and $\mathrm{CD} 34^{+} \mathrm{SCs}$, along with their immunomodulatory functions, presumably tightly regulated by epigenomic modifications and gene regulatory networks, is less well defined.

Although, the initial priming of the adaptive immune response heavily relies on antigenpresenting DCs, the intrinsic microenvironment of the respective tissue-draining LN and its SC compartment, greatly influences this process. Particularly, the heterogeneous population of FRCs has been shown to shape the adaptive immune responses by providing survival molecules such as IL-7 and BAFF, ${ }^{16,17}$ or readily upregulating iNOS upon IFN $\gamma$ signaling, thereby limiting the expansion of pro-inflammatory $\mathrm{T}$ cells and globally suppressing aberrant priming of adaptive immune cell differentiation. ${ }^{18,19}$ Distinct localization of FRC subsets, such as Cxc19producing FRCs, can mitigate effective migration of $\mathrm{Cxcr}^{+}$cells, including memory $\mathrm{CD}^{+} \mathrm{T}$ cells, during the course of antiviral immune responses. ${ }^{12,20}$ In addition to these immuno-modulatory functions inherent to any LN, the local SC compartment also tissuespecifically shapes the migratory and effector $\mathrm{T}$ cell properties. ${ }^{11,21,22}$ Despite the detailed understanding of prenatal LN development, little is known about which transcriptional regulators impinge postnatally on the presumably common pool of mLTos to give rise to the heterogeneous population of non-endothelial $\mathrm{SCs}^{23}$ and their immune-modulatory 
potential. ${ }^{11,22,24}$ Although multiple mechanisms through which mesenchymal SCs modulate the LN microenvironment have been identified, the underlying transcriptional regulators and epigenomic framework defining distinct functional SC responses from birth and throughout adulthood are only incompletely understood..$^{25,26}$

Here, we have mapped the location-specific epigenomic landscape of non-endothelial SCs encompassing both FRCs and CD34 ${ }^{+}$SCs. We identified location-specific, epigenetically modified genomic loci, and based on motif enrichment analysis together with gene expression profiling, delineated steady-state expression signatures poising peripheral skin-draining LN (pLN) SCs for pro-inflammatory responses. Furthermore, by obtaining the first developmental scRNA-seq profiling dataset following mesenteric lymph node ( $\mathrm{mLN}) \mathrm{SC}$ development from birth throughout adult life, we also observed the postnatal segregation of mesenchymal progenitors and confirmed their distinct localization within the developing mLNs. We further outlined the developmental trajectory of the mLNs' SC compartment and mapped subsetspecifically expressed transcription factors (TF) to distinct expanding SC populations, which shape the epigenomic landscapes across CD $34^{+}$SCs and FRCs. Finally, using lentiviral overexpression, we uncovered $\operatorname{Irf} 3$ as a key transcriptional regulator driving mesenchymal stem cell differentiation towards $\mathrm{Cxc19}^{+} \mathrm{FRCs}$.

Our data constitute a comprehensive transcriptional map of SC development in mLNs. More precisely, we characterize the transcriptional and epigenomic landscape of non-endothelial LNSCs, providing a valuable resource to delineate transcriptional regulators that govern the developing LN and thereby permanently shape adaptive tissue-specific immune responses. 


\section{Results}

\section{Location defines the epigenomic landscape of non-endothelial LNSCs}

Previous studies underlined the immuno-modulatory functions of LNSCs. ${ }^{27}$ We thus aimed to delineate epigenomic modifications that underlie the immuno-modulatory function of LNSCs. To this end, we performed whole-genome bisulfite sequencing (WGBS) and assay for transposase accessible chromatin sequencing (ATAC-seq) to identify CpG methylation and genomic accessibility, respectively, of $\mathrm{CD} 31^{-} \mathrm{Pdpn}^{+}$non-endothelial SCs isolated by fluorescence-activated cell sorting (FACS) from mLNs and pLNs originating from adult mice housed under specific pathogen-free (SPF) or germ-free (GF) conditions. We were able to determine the methylation status of $88.2 \%$ of the $2.19 * 10^{7} \mathrm{CpGs}$ and, using Bsmooth, ${ }^{28}$ identifying 1532 non-overlapping differentially methylated regions (DMRs) across all pairwise comparisons. Importantly the vast majority of the DMRs were location-dependent (Fig. 1A), whereas only 16 and 17, were commensal-dependent for pLN and mLN, respectively (Supplementary Table 1). Over 390 DMRs, located in the proximity of the transcription start site (TSS), were annotated to 286 genes, including microenvironmental mediators (e.g. Cxcll3, Gdf6, Sfrp5), immuno-modulatory enzymes (e.g. Aldh1a2, Ptgis), ${ }^{29-32}$ and TFs (e.g. Isll, Hoxd1, Meis 1, Meis2, Nkx2-3, Tcf4, Foxn2), potentially impinging on transcriptional regulation (Fig. 1B-C). ${ }^{33}$

We then proceeded to obtain a global overview of chromatin accessibility. Peaks were called per sample and merged to obtain common open genomic regions across all conditions comprising a total of 42,434 peaks (Supplementary Table 2) As expected, we identified a substantial number of 5327 differentially accessible regions (DARs) between mLN-SPF and pLN-SPF, whereas absence or presence of microbiota only marginally influenced the accessibility profile of non-endothelial SCs for both mLN and pLN, corroborated by the low number of detected DARs (11 and 64, respectively; Fig. 1D). Gene ontology (GO) analysis of the location-dependent DARs revealed that mLN SCs are responsive to BMP signalling and negatively regulate inflammatory processes (Fig. 1E). Surprisingly, pLN SCs did not show increased chromatin accessibility for pro-inflammatory mediators (Fig. 1E), which is in contrast to the previously made observations at the transcriptional level. ${ }^{11,34}$

Altogether, these data implicate that the tissue-specific location of LNs strongly influence the epigenomic landscape of non-endothelial SCs, thereby potentially contributing to TF-controlled transcriptional programs. 



Figure 1. LN location defines the epigenomic landscape of non-endothelial SCs. CD45 ${ }^{-}$D24-CD31-Pdpn ${ }^{+} \mathrm{SCs}$ were isolated from mLNs and $\mathrm{pLNs}$ of GF or SPF mice, and WGBS (A-C) or ATAC-seq (D-E) analyses were performed. (A-C) DMRs were identified in colonization- (SPF vs. GF) and location-dependent (mLN vs. pLN) pairwise comparisons. Scale bar depicts the extent of methylation with $100 \%$ being fully methylated and $0 \%$ being non-methylated. (A) The heatmap represents the mean methylation of significant DMRs within the promotor region of 284 genes. (B) Heatmaps represent CpG methylation of exemplary DMRs. The distance from the TSS is indicated and the size of genomic loci denoted in base-pairs (bp). (C) The heatmap represents the mean methylation of significant DMRs within the promotor region of TFs. (D-E) DARs were identified in colonization- (SPF vs. GF) and location-dependent (mLN vs. pLN) pairwise comparisons. (D) Volcano plots of mean ATAC-seq FPKM comparing indicated samples. The number of DARs (top) and genes (bottom) is indicated per comparison. (E) GO analysis of biological processes of genes with location-dependent DARs. The numbers denote GO identifiers. ATAC-seq, assay for transposase accessible chromatin sequencing; DAR, differentially accessible region; det, detection; DMR, differentially methylated region; GF, germ-free; GO, gene ontology; met, metabolism; neg, negative; pos, positive; reg, regulation; SC, stromal cell; sig, signalling; SPF, specific pathogen-free; TF, transcription factor; TSS, transcription start site; WGBS, whole-genome bisulfite sequencing. 


\section{Differential accessibility delineates location-dependent regulators of persistent SC function}

The observed discrepancy between gene-associated DARs and previously published gene expression signatures particularly for non-endothelial $\mathrm{pLN} \mathrm{SCs}^{11,34}$ prompted us to further dissect transcriptional and epigenomic co-regulation. In order to compare both transcriptome and chromatin accessibility, we obtained RNA-seq data of $\mathrm{CD}^{-} 1^{-} \mathrm{Pdpn}^{+}$non-endothelial SCs isolated using FACS from mLNs and pLNs originating from adult mice housed under SPF conditions. We then correlated location-dependent differential expression with the respective chromatin accessibility within the promotor region on a per-gene basis, which allowed us to divide genes into three distinct groups (Fig. 2A). The first group includes genes whose transcriptional activity correlates with chromatin accessibility, with 207 and 183 for mLN SCs and pLN SCs, respectively. These genes comprise $24 \%$ of the differentially expressed genes (DEGs) and include genes such as Nkx2-3, Ccl20 and Cxcl9, known for their locationdependent differential expression. ${ }^{11,34}$ The second group contains $43 \%$ of the regulated genes that are differentially accessible, but not differentially expressed, potentially composed of elements that respond to cellular activation. Interestingly, 296 genes for mLN SCs and 255 genes for pLN SCs are differentially expressed, but have no associated DARs, indicating that these genes are regulated by TFs that exploit already accessible chromatin and that we define here as active TF regulated (Fig. 2A). We performed GO analysis for biological processes on the inducible gene modules or active TF regulated genes for mLN SCs and pLN SCs, and identified particularly for the genes associated with active TFs for pLN SCs a substantial enrichment of GO terms associated with elevated immune responses including neutrophil chemotaxis and response to cytokine (Fig. 2B-C). Interestingly, within mLN SCs, GO terms indicative of immune suppression were enriched, including the negative regulation of $N f k b$ signalling (Fig. 2B-C).

Next, we aimed to identify the TFs responsible to alter expression without modifying chromatin accessibility under steady-state conditions. To this end, we identified over-represented TF binding sites (TFBS) and their putative TFs, for the inducible gene modules and active TF regulated genes within accessible genomic loci of the respective genes. As expected, a substantial number of TFs, namely Klf5, Jun, Atf3, Batf, Bach2, Sp1, Atf1, Nf1 and Jund were enriched in at least five out of six gene loci sets indicating their general involvement in shaping the function of non-endothelial SCs (Fig. 2D, Supplementary Fig. 1). Surprisingly, only the inducible gene module for mLN SCs and the active TF regulated module for pLN SCs showed unique over-representation of TFBS and putative TFs (Fig. 2D). Therefore, we assessed which of the putative TFs identified from the genomic loci within pLN SCs are differentially expressed, and determined Irf1, Irf5, Irf8, Nfib and $N f k b 2$ to potentially contribute to the increased transcription of pro-inflammatory genes in pLN SCs under steady-state conditions (Fig. 2E), whereas E2fl and Isll were solely identified from inducible gene modules of mLN SCs (Fig. 2F).

Together with a large number of shared TFs, the observed TFBS enrichment patterns suggest that non-endothelial SCs contain a location-dependent epigenomic landscape, allowing distinct TF regulation in $\mathrm{pLN}$ and $\mathrm{mLN}$. Our results moreover uncovered a defined set of TFs, including Irf1, Irf5 and Irf8, that drive increased expression of pro-inflammatory cytokines in pLN nonendothelial SCs independent of chromatin accessibility and pinpoint uniquely active TFs in $\mathrm{pLN}$ and $\mathrm{mLN}$. 

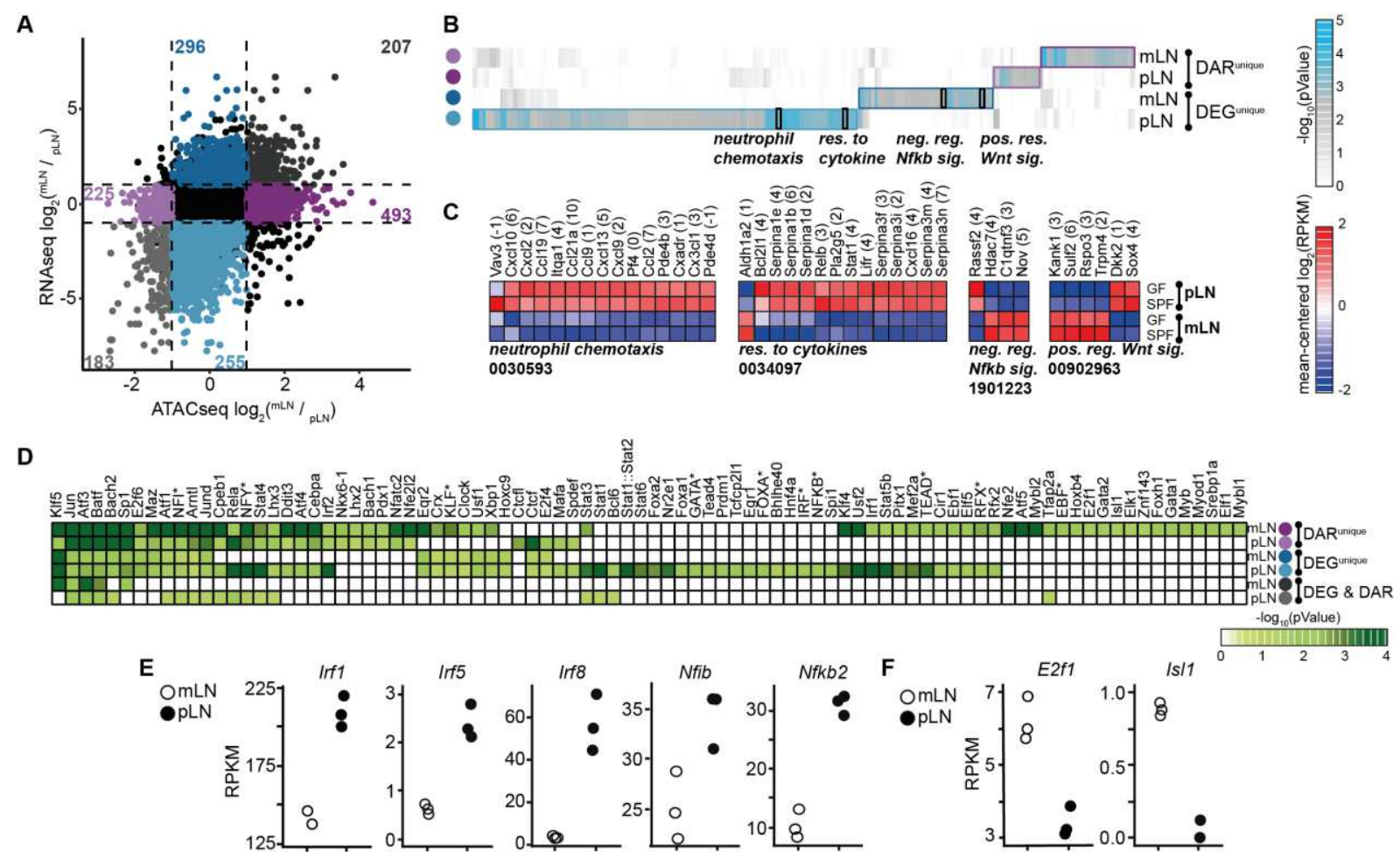

Figure 2. Accessible and actively expressed TFs promote pro-inflammatory gene expression profile of pLN non-endothelial SCs. CD45 ${ }^{-}$D24 ${ }^{-} \mathrm{CD} 31^{-} \mathrm{Pdpn}^{+} \mathrm{SCs}$ were isolated from $\mathrm{mLNs}$ and pLNs of SPF mice. Subsequently, RNA-seq (A-F) or ATAC-seq analyses (A-B, D) were performed. DEGs and DARs were identified in $\mathrm{mLN}$ vs. pLN pairwise comparisons. (A) Colored numbers in the scatterplot represent the number of genes with DAR and/or differential expression. Only genes with accessible loci within the promotor region were included in the analysis. On the $\mathrm{x}$-axis, $\log _{2}(\mathrm{FC})$ of accessibility per DAR is plotted and on the $y$-axis, the $\log _{2}(\mathrm{FC})$ of gene expression for the comparison of mLN vs. pLN. (B) GO analysis of biological processes of DEGs and/or genes with at least one DAR. (C) Heatmaps represent the expression of all DEGs within the GO groups highlighted in (b). The numbers denote GO identifiers. (D) The enrichment of known TFBS motifs for each of the quadrants in (A) was utilized to identify TFBS over-represented in the accessible chromatin. The heatmap represents the p-value for enriched TFBS and its corresponding putative TF(s). (E) The scatterplots depict differentially expressed TFs that were uniquely identified from the accessible loci of the 255 DEGs for pLN. (F) The scatterplots depict differentially expressed TFs that were uniquely identified from the accessible loci of the 493 DEGs for mLN. ATAC-seq, assay for transposase accessible chromatin sequencing; DAR, differentially accessible region; DEG, differentially expressed gene; det, detection; FC, foldchange; SC, stromal cell; GO, gene ontology; neg, negative; pos, positive; reg, regulation; res, response; sig, signalling; TFBS, transcription factor binding site; TF, transcription factor. 


\section{Developmental age constrains cellular and functional composition of the mLN SCs}

We hypothesized that the observed differences across the epigenomic landscapes of nonendothelial SCs derive from epigenomic modifications that were obtained during early postnatal development. It is widely accepted that substantial epigenomic modifications occur during the transition of progenitors to fully differentiated cells. ${ }^{35,36}$ As LNs undergo rapid postnatal expansion, we first aimed to dissect changes in SC composition along $\mathrm{mLN}$ development.

To this end, we resected mLNs from mice at early postnatal age (day 0/1, D0), the early (D10) and late (D24) juvenile stages, adult age (D56), as well as old age (D300), and obtained FACSpurified $\mathrm{CD}^{-} 5^{-} \mathrm{CD} 24^{-}$cells. We performed single-cell (sc)RNA sequencing and gathered transcriptomes of 15,925 cells with comparable sequencing depth $(55,000-98,000$ mean reads per cell) across the different time points. Initially, we used transcriptional signatures for mLN $\mathrm{SCs}^{11}$ and the canonical marker for endothelial cells Pecaml (Supplementary Fig. 2A) to remove endothelial cells and perivascular cells $(\mathrm{PvC})$ from the analysis, after alignment of samples using diagonal canonical correlation analysis (Methods). Additionally, we observed and excluded cells that were disproportionately represented in D0/1 mLNs, expressing significantly higher levels of the growth factors Igfl and Igf2 as well as the extracellular matrix protein Mfap4, indicative of adjacent tissue fibroblasts (Supplementary Fig. 2B-C, Supplementary Table 3). To obtain an unbiased picture, we re-embedded 5,658 nonendothelial SCs across all five developmental stages. Twelve transcriptional clusters harboring unique functional properties were identified based on DEGs, GO analysis and previously published signatures (Fig. 3A, Supplementary Fig. 2D-E, Supplementary Table 4). ${ }^{1,12}$ These clusters were broadly separated into $\mathrm{CD} 34^{+} \mathrm{SC}$, including CD34 ${ }^{+(\mathrm{CD} 248+)}, \mathrm{CD} 34^{+(\mathrm{Ackr} 3+)}$, $\mathrm{CD} 34^{+(\text {Aldh1a2+)}}$, metabolically active (mFRC), $\mathrm{Il}^{+(\mathrm{Cxcl1}+)} \mathrm{FRC}, \mathrm{Cxcl}^{+} \mathrm{FRC}, \mathrm{Inmt}^{+} \mathrm{FRC}$, Inmt $^{+(\mathrm{Cxcl} 12+)}$ FRC and Ccl19 ${ }^{+(\mathrm{II7+})}$ FRC, mesothelial-like (Meso), LTo-like and progenitor-like (Prog) subsets (Fig. 3A). All identified clusters were variably represented along postnatal mLN development (Fig. 3B-C), with particularly LTo-like and Prog subsets being over-represented at D0 and D10. As expected, LTo-like SCs highly expressed Cxcl13, Tnfsf11 and Madcam1 (Fig. 3D-E). ${ }^{2,3,9}$ Interestingly, we observed an additional highly proliferating subset, here termed postnatal progenitors (Prog; Fig. 3C-E). The progenitor subset could further be subdivided into two distinct populations (Fig. 3F). Prog ${ }^{\text {Cxcl13+ }}$ cells expressed significantly higher levels of canonical markers for LTo-like cells including Madcaml and Ccl19, indicative of a close relation to previously described LTos and their propensity to function as progenitors during LN development. ${ }^{37}$ Surprisingly, we identified an additional subset, here termed Prog ${ }^{\mathrm{CD} 34+}$ which showed higher expression for several collagens (Col3a1, Col6a2 and Colla2), as well as genes involved in regulating Bmp-signalling including Gpc3 and Crip (Fig. 3F). ${ }^{38}$

We then aimed to identify the localization of the respective putative progenitor populations within the developing LNs by utilizing RNAscope on neonatal $\mathrm{mLN}$ tissue slices. Initially, we confirmed that i) the RNA integrity of D0 mLN remained intact after resection by staining for a mix of ubiquitously expressed genes (Polr2a, Ubc, Ppib) as a positive control (Supplementary Fig. 3A) and ii) the majority of $\mathrm{Ccll}$ ) and $\mathrm{Cxcll3}$ expressing cells were spatially separated from $C d 34^{+}$cells at this stage of postnatal mLN development (Supplementary Fig. 3B-C). Further, we utilized cyclin-dependent kinase 1 ( $C d k l)$, a key kinase at the transition to the S-phase, which is highly expressed in both of the two putative progenitor populations, as a proxy to pinpoint progenitor cells. We readily identified capsular $C d 34$ and $C d k 1$ expressing non-endothelial $C d 31^{-}$cells (Fig. 3G). Importantly, $C d 34^{+} C d k 1^{+}$ cells were neither present within the cortical nor paracortical areas, but could be identified within adjacent tissue (Supplementary Fig. 3D). As expected, we furtheron detected $\mathrm{CdkI}^{+} \mathrm{Cxcll}^{+}$cells, ${ }^{3,4}$ predominantly in the developing cortex (Fig. 3H). 
Together, these data delineate the postnatal expansion of the heterogeneous non-endothelial SC compartment and identify two putative postnatal progenitor cell populations that are distinctly positioned within the developing LN. 
A
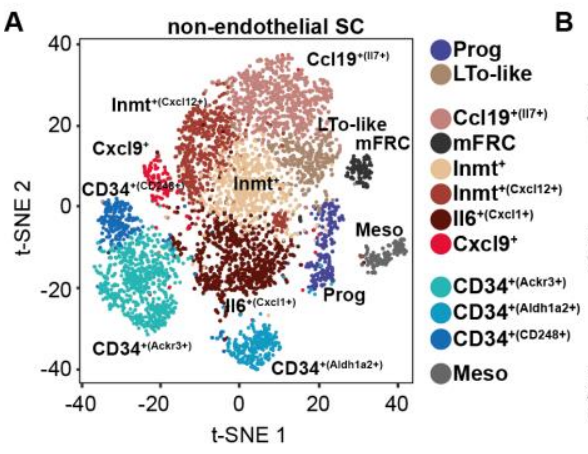
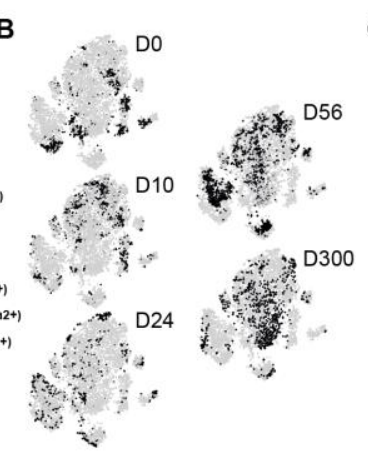

C
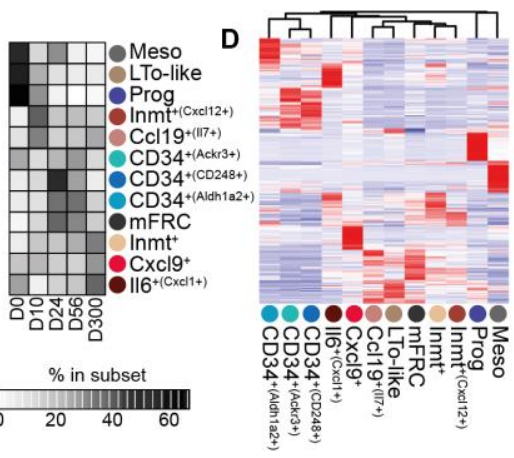

E Aldh1a2 $\left.\right|_{0} ^{3}$ Bst1 $\left.\right|_{0} ^{3}$ CD
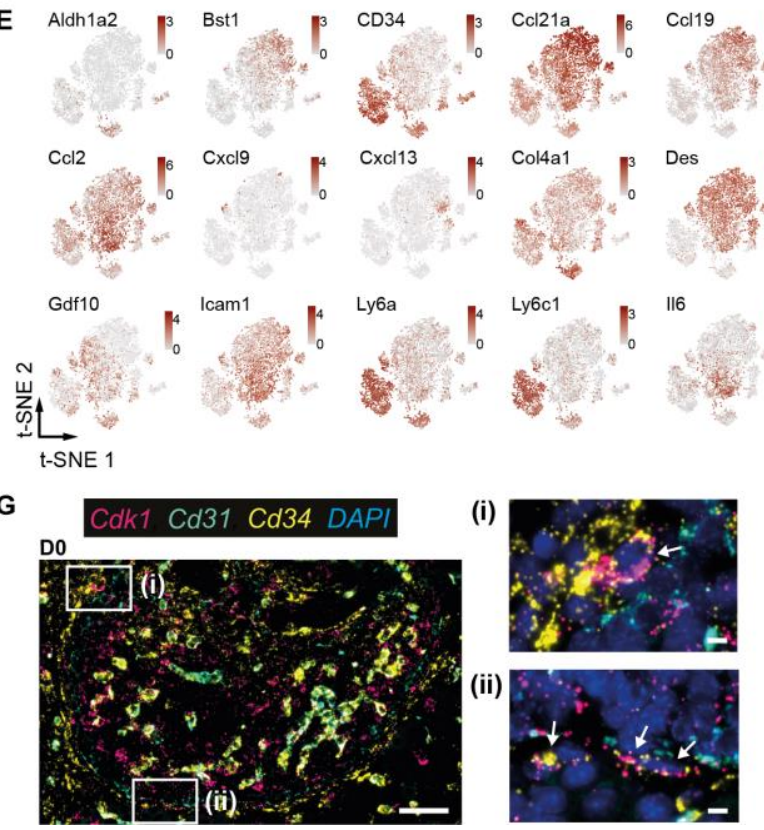

(i)

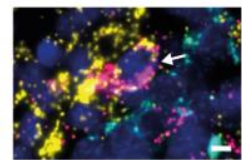

(ii)

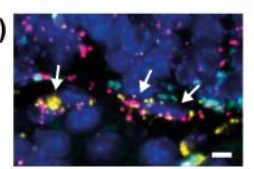

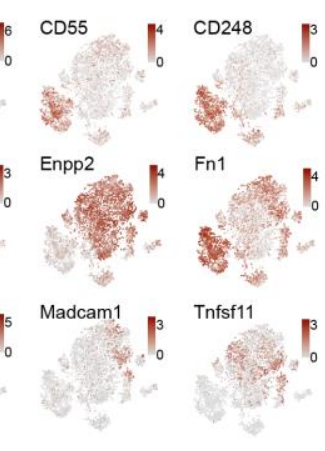

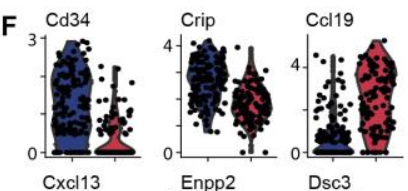
Enpp2
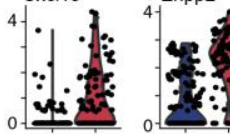

Madcam1

Peg3



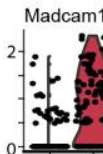

H

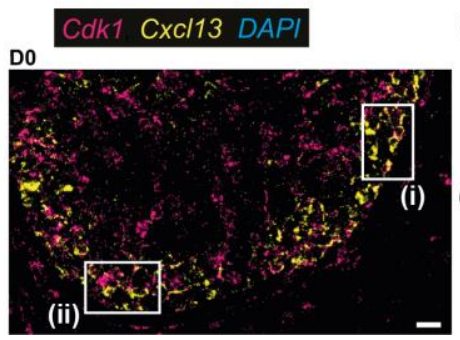

(ii)
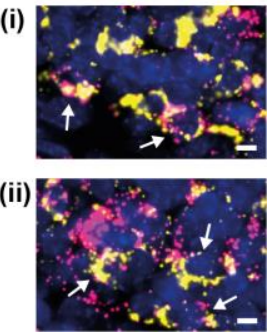

Figure 3. Postnatal ontogeny of non-endothelial mLN SCs. CD45CD24 cells were isolated from mLNs of day $0,10,24,56$ and 300 old SPF-housed mice and subjected to scRNA-seq. Non-endothelial SCs were identified as non-LECs, non-BECs and non-PvCs. (A) t-SNE plot of merged SCs across ages showing cluster segregation. (B) t-SNE plot of SCs from each age. (C) The heatmap represents the percentage of cells in each subset across time points normalized to cell number. (D) Hierarchical clustering of subsets based on the mean expression of the top 40 DEGs per subset. (E) t-SNE plot colored for expression of segregating genes. (F) Violin plot of DEGs for subsets identified among putative postnatal progenitors. (G-H) Sections $(3 \mu \mathrm{m})$ of D0 neonatal mLN were stained with the indicated RNAscope probes and imaged by fluorescence microscopy. Nuclei were counter-stained with DAPI (blue). Images represent an overview of $\mathrm{mLN}$ anlagen with "white squares" indicating regions of interest. (i-ii) Images represent zoom-ins of respective regions of interest. Arrows indicate marker co-expression per cell. Representative tissue sections $\mathrm{n}=2-3$. (G) Capsular positioning of $\mathrm{Cd} 34^{+} C d k \mathrm{I}^{+}$cells. Overview (scale bar $=50 \mu \mathrm{m}$ ) and zoom-in (scale bar $=5 \mu \mathrm{m}$ ) for RNA probes specific to Cdkl, Cd31 and Cd34. (H) Cortexlocalized $\mathrm{Cxcll}^{+} \mathrm{Cdkl}^{+}$cells. Overview (scale bar $=20 \mu \mathrm{m}$ ) and zoom-in (scale bars $=5 \mu \mathrm{m}$ ) for RNA-probes specific to $C d k 1$ and $C x c l 13$. BEC, blood endothelial cell; LEC, lymphatic endothelial cell; LTo-like, lymphoid tissue organizer like cell; Meso, mesothelial-like cells; mFRC, metabolically active FRC; Prog, postnatal progenitor; PvC, perivascular cell; SC, stromal cell. 
Distinct progenitors establish the non-endothelial SC compartment rapidly after birth Previous studies showed that postnatal expansion of non-endothelial SCs within the LN relies on the progenitor potential of LTo cells, differentiating into various SC subsets. ${ }^{14,39,40}$ Additionally, progenitors with LNSC potential have been shown to reside in the $\mathrm{CD} 34^{+}$perivascular niche of multiple adult organs. ${ }^{10}$ Therefore, we aimed to elucidate whether LTo-like, putative Prog ${ }^{\mathrm{Cxcl} 13+}$ and Prog ${ }^{\mathrm{CD} 34+}$ progenitors observed at early postnatal mLN development have the molecular potential to give rise to $\mathrm{CD} 34^{+} \mathrm{SC}$ and FRCs in the expanding neonatal $\mathrm{mLN}$.

We initially constructed a trajectory using all non-endothelial SCs, and observed two distinct sets of branches evolving along physiological development (Supplementary Fig. 4A), underscored by expression of key marker genes identifying progenitors at distinct, presumably early stages of development (Supplementary Fig 4B). ${ }^{27,37,41}$ We therefore re-embedded cells situated along the CD34 ${ }^{+}$SC or FRC trajectory (Supplementary Fig. 4C) and employed pseudo-time mapping using Monocle $2 .{ }^{42}$ As expected, early developmental time points corresponded to pseudotemporal branches at the starting point of the trajectory (Fig. 4A-B). Importantly, already at D24, CD34 ${ }^{+} \mathrm{SCs}$ were distributed along the complete pseudotemproal space, whereas only minor changes in the distribution of cells across pseudotime were observed beyond D56, indicating that main non-endothelial SC differentiation is established shortly after weaning (Fig. 4A-B). While Ccl19 and Ptgis were constitutively expressed over the developmental trajectory, subset-defining genes including Aldhla2 and Il6 were predominantly expressed at distinct branches of the $\mathrm{CD} 34^{+} \mathrm{SC}$ or FRC trajectories, respectively (Fig. 4B-C), indicative of developmental segregation for each subset of $\mathrm{CD} 34^{+} \mathrm{SCs}$ and FRCs, culminating in distinct functional subsets (Fig. 4D). Importantly, $\mathrm{Cxcll} 3$ was expressed highest early during development in FRCs (Fig. 4C). As expected, pseudotime starting points of both the CD $34^{+}$SC

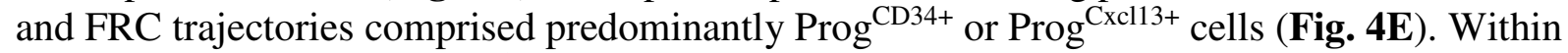
the FRC trajectory, Prog ${ }^{\mathrm{Cxcl} 13+}$ cells pseudotemporally aligned with the LTo-like ones, indicating that these cells are of similar origin, giving rise to two major branches of differentiation that correspond to different subsets of FRCs, namely $\mathrm{Ccl}^{+1} 9^{+(\mathrm{II} 7+)}$ and $\mathrm{Il}^{+(\mathrm{Cxcl1}+)}$ (Fig. 4E-F). Distinct differentiation paths were underscored by an increased expression of Tnfsf13b, Il33, Ccl19 and Tgfbi for Ccl19 ${ }^{+(117+)}$, and Cxcll and Il6 for $\mathrm{Il}^{+(\mathrm{Cxcl1+})}$ subsets along pseudotemporal development (Fig. 4C, Supplementary Fig. 4D).

In line with the FRC trajectory, the $\mathrm{CD} 34^{+} \mathrm{SC}$ development initiated from $\mathrm{Prog}^{\mathrm{CD} 34+}$ cells, branching into two major axes consisting predominantly of either CD34+(CD248+) or CD34+(Aldh1a2+) (Fig. 4E-F). As expected, a small proportion of $\mathrm{CD}^{+} 4^{+} \mathrm{SCs},{ }^{2,10}$ was already in place at D0, rapidly expanding at D10 and D24 (Fig 4B). As anticipated, based on the heterogenous composition of the mLN's non-endothelial SC compartment, the main terminal branches showed distinct expression profiles. While the $\mathrm{CD} 34^{+(\mathrm{CD} 248+)}$ branch expressed higher levels of Ptgs1 and Ptgis enabling prostacyclin synthesis (Fig. 4C, Supplementary Fig 4D), the CD34 ${ }^{+(\text {Aldh1a2+) }}$ branch expressed higher levels of Coll5al and Vtn together with Fgf7 and Gdf10 potentially generating a specific cell adhesion environment (Supplementary Fig. 4D). Surprisingly, cells of the $\mathrm{Cxcl}^{+}$subset were distributed similarly between FRCs and $\mathrm{CD} 4^{+} \mathrm{SCs}$ and were not over-represented at a specific terminal branch, neither in the FRC nor the $\mathrm{CD}_{3} 4^{+} \mathrm{SC}$ trajectory (Fig. 4D). The dispersion of the $\mathrm{Cxcl}^{+}$subset across the two main subsets and the trajectory is underscored by the unique expression of $L y 6 a$, also a core feature of CD $34^{+}$SCs, but the lack of consistent $C d 34$ expression (Fig. 3E).

In sum, the pseudotemporal dissection of the non-endothelial SC compartment indicates that postnatal expansion of the main populations of $\mathrm{mLN} S \mathrm{Ss}$ is driven by two proliferating 
progenitor populations, both of which are already postnatally set on a defined FRC or CD $34^{+}$SC differentiation trajectory. 


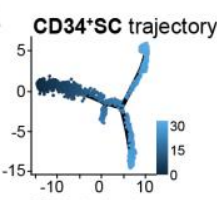

$$
\text { B }
$$<smiles>CC(C)C(C)C(C)C(C)C(C)C(C)C(C)C(C)C</smiles><smiles>CC(C)CC(C)CC(C)C</smiles>

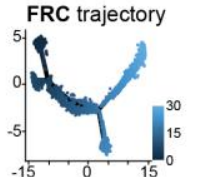<smiles>CC(C)C(C)C(C)C(C)CBr</smiles>

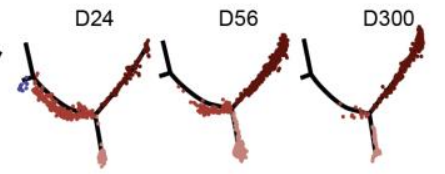

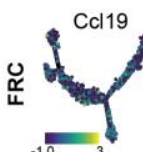<smiles>CCCC(C)CC(C)C</smiles>

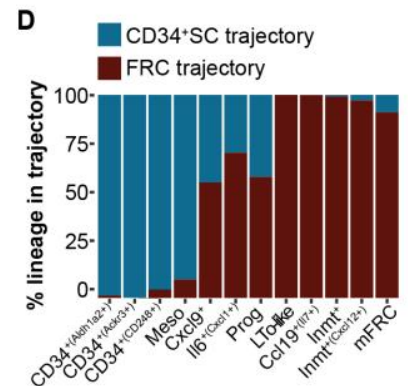

E

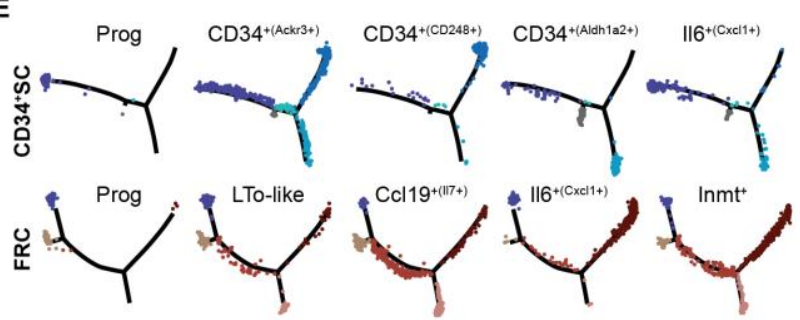



Figure 4. Distinct postnatal progenitors give rise to FRCs and CD34 ${ }^{+}$SCs. CD45-CD24- cells were isolated from mLNs of day 0, 10, 24, 56 and 300 old SPF-housed mice and subjected to scRNA-seq. Non-endothelial SCs were identified as non-LECs, non-BECs and non-PvCs. (A) Pseudotime ordering of FRCs and CD34 ${ }^{+}$SCs. (B) Pseudotime trajectories superimposed with cells per time point. (C) Gene expression on pseudotime trajectories. (D) Bar graph depicts the proportion of cells from the indicated subset within the FRC or CD34 ${ }^{+} \mathrm{SC}$ trajectory. (E) Cells per cell subset across branches superimposed on pseudotime trajectories. (F) The heatmap represents the percentage of cell subsets across branches normalized to cell number. BEC, blood endothelial cell; FRC, reticular fibroblastic stromal cell; LEC, lymphatic endothelial cell; mLN, mesenteric lymph node; PvC, perivascular cell; SC, stromal cell; SPF, specific pathogen-free. 


\section{TFs shape the subset-specific epigenomic landscape early during ontogeny}

TFs guide epigenomic modifications to respective target loci, particularly during differentiation processes. ${ }^{43-45}$ We hypothesized that most TFs that drive the differentiation of the two main non-endothelial SC subsets from progenitors to fully functional CD34 ${ }^{+} \mathrm{SCs}$ or FRCs, as inferred from footprinting analyses of the accessible chromatin, should also be dynamically expressed along mLN development. To assess this, we explored TF binding motif enrichment in the epigenomic landscape of mLN SCs and investigated which TFs are expressed at key branching points of the $\mathrm{FRC}$ and $\mathrm{CD} 34^{+} \mathrm{SC}$ differentiation trajectories.

To this end, we combined the over-represented TFBS and their putative TFs, within accessible genomic loci (Fig. 2D) with the trajectoral expression of all TFs detected in the developmental scRNA-seq analysis of CD34 ${ }^{+}$SCs and FRCs (Fig. 4). In total, 30\% of the TFs, identified from the accessible chromatin, were differentially expressed over the developmental trajectory, of which 7 and 13 TFs were uniquely differentially expressed at the key branching point for CD $34^{+}$SCs and FRCs, respectively (Fig. 5A-C). Particularly, the commonly over-represented TFs (Fig. 2D) are expressed at the key branching point of both $\mathrm{CD} 34^{+} \mathrm{SC}$ and FRCs (Fig. 5A-B), including Atf3, Egrl, Egr2, Irfl, Jun, Jund and Klf9, indicating that these TFs shape the general course of chromatin accessibility of differentiating SCs (Fig. 5A-C, Supplementary Fig. 4E). Importantly, $40 \%$ of the TFs that were differentially expressed and motif enriched TFs between pLN SCs and mLN SCs are shared between both CD $34^{+}$SCs and FRCs, indicating their general requirement in shaping the epigenomic landscape and function of these two non-endothelial main SC subsets (Fig. 5C).

We next utilized dynGENIE $3^{46}$ to identify key TFs that potentially impinge upon the differentiation of the cellular components of the expanding $\mathrm{mLN}$, and constructed a gene regulatory network of dynamically regulated genes encompassing TFs for each of the two terminal branches for both FRCs and CD $34^{+}$SCs. In line with the branch-point-based analysis, we observed that $23 \%$ of the dynamically regulated TFs were co-regulated consistently across FRCs and $\mathrm{CD}_{34}{ }^{+} \mathrm{SCs}$, including Nfia, Egr2 and Ebf2 (Fig. 5D-E). Despite the substantial proportion of common TF denominators, particularly for the IRF and KLF/SP TF family, a noticeable number of TFs were uniquely regulated along the trajectories. While Irfl and Klf4/9/13 emerged for CD34 ${ }^{+} \mathrm{SCs}$, Irf3 and Klf6/7/11 were solely identified for FRCs (Fig. 5C-E), suggesting dissimilar modulatory paths during the development of these two distinct non-endothelial SCs.

In summary, by combining branch-point-based differential expression together with identification of dynamically co-regulated gene networks, we were able to identify TFs that are potentially decisive in postnatally shaping the differentiation of $\mathrm{CD}_{3} 4^{+} \mathrm{SCs}$ or FRCs. 
A

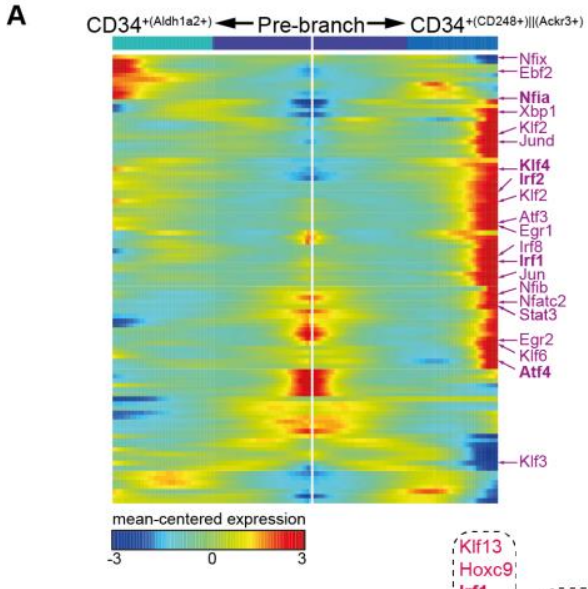

B

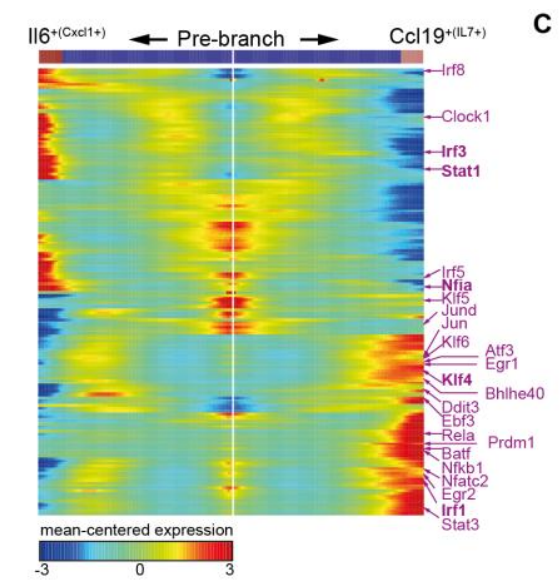

E $\square \| 6^{+(C x d 1+)}$



Figure 5. TFBS accessibility aligns with dynamically regulated TFs to shape differentiation and function of key mLN SC subsets. (A-B) Heatmap showing expression of TFs over pseudotime involved in the differentiation of (A) CD $34^{+}$SCs and (B) FRCs. TFs highlighted in "pink" are derived from TFBS enrichment analyses of mLN SC DARs. (C) The Venn diagram depicts the overlap of accessibility-derived TFs. TFs upregulated per subset/branch are denoted in "black". (D-E) Gene regulatory networks based on genes that are dynamically coexpressed along the developmental trajectory, ordering cells within each "branch" per age (see Figure 4). The size of the node is proportional to the node outdegree. TFs inferred from their respective TFBS in accessible chromatin regions (see Figure 2) annotated to the respective node cohort in „,pink“| DARs and "black” | SC-associated genes. The color of the edges signifies the terminal branch from which the edge was derived. (D) depicts CD $34^{+}$SCs. (E) depicts FRCs. DAR, differentially accessible region; det, detection; DMR, differentially methylated region; FRC, reticular fibroblastic stromal cell; mLN, mesenteric lymph node; SC, stromal cell; TF, transcription; TFBS, transcription factor binding sites. 


\section{Members of the IRF transcription factor family contribute to the differentiation of CD34 $^{+}$SCs and FRCs}

By combining orthogonal analyses of TFBS enrichment and gene expression along mLN development, we were able to identify a concise assembly of TFs that are putatively involved in the differentiation of non-endothelial SC subsets. We therefore hypothesized that these TFs should be able to drive the differentiation from multipotent progenitor cells towards a cell-type or state-specification resembling ex vivo profiled SC subsets.

To this end, we utilized a well-established TF-overexpression model based on lentiviral integration, puromycin selection and timed doxycycline-driven overexpression ${ }^{47}$ in a murine C3H10T1/2 mesenchymal stem cell like (MSC) line. ${ }^{48}$ We focused on TFs that were either identified for both CD34 ${ }^{+}$SCs and FRCs (e.g. Atf4, Nfia, Irf1), only for CD34 ${ }^{+}$SCs (e.g. Irf2, Cebpa), or only for FRCs (e.g. Irf3, Stat1). We also included Myc, as LTos have myofibroblastic features, ${ }^{9}$ and Fos as it is widely expressed across all non-endothelial SC (Supplementary Fig. 2E). ${ }^{11,12}$ We initially assessed cellular morphological changes of differentiated MSCs and observed a broad range of morphologies, including upregulation of lipid droplet formation for $\mathrm{Nfia}^{49}$ and distinctive fibroblastic features for the majority of overexpressed TFs, including Irfl, Irf3 and Myc (Fig. 6A). We then assessed the gene expression profile of induced MSCs by performing bulk 3'RNA-seq (BRB-seq) at the terminal differentiation time point, 12 days post doxycycline-driven overexpression. ${ }^{50}$ To compare the extent of matching expression signatures for each of the overexpressed TFs with the endogenous subset-specific signatures, we calculated the cumulative Z-score for the Top 100 DEGs for each of the endogenously identified SC subsets (Supplementary Table 4). As expected, $M y c$ overexpression drove an LTo-like expression signature, consistent with a myofibroblastic origin (Fig. 6B). ${ }^{9}$ The most striking overlap of the endogenous expression signature was observed for Irf3 overexpression, supporting the expression signature of DEGs identified for the $\mathrm{Cxcl}^{+}$FRCs, which was further corroborated on a per cell basis when calculating the cZscore from the Irf3-driven overexpression (Fig. 6C). Furthermore, Irf3 overexpression supported the upregulation of $\mathrm{Cxcl}^{+} \mathrm{FRC}$ subset-associated genes, including several interferon-induced proteins (e.g. Ifitl, Ifit3, Ifit3b) and antiviral response elements (e.g. Is 15 , Oasl2) above the expression levels detected for any of the overexpressed TFs including Irfl and Irf2 (Fig. 6D).

Based on the comprehensive epigenomic and gene expression analyses of mLN SC development, we identified TFs that may shape the postnatal immuno-modulatory specialization of LNSCs. By overexpressing a selected set of delineated TFs, we validated Irf3 to distinctly promote the differentiation from MSCs to a cell phenotype that molecularly resembles $\mathrm{Cxcl}^{+}$FRCs. 
A

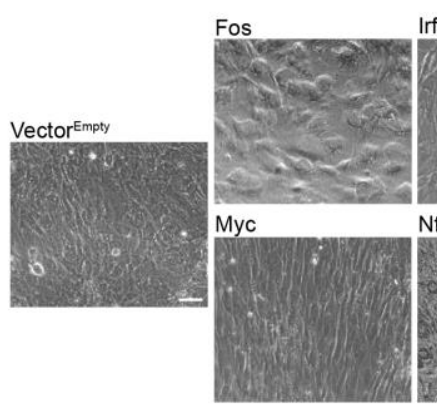

Iff1



Iff3

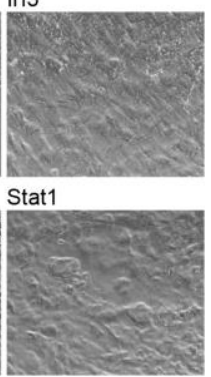

B

CZscore
ex vivo DEGs per subset

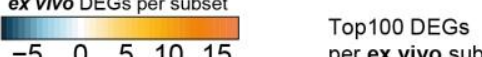

per ex vivo subset

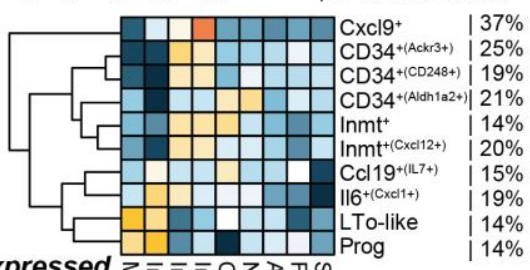

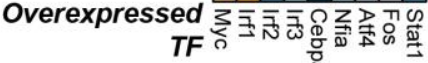
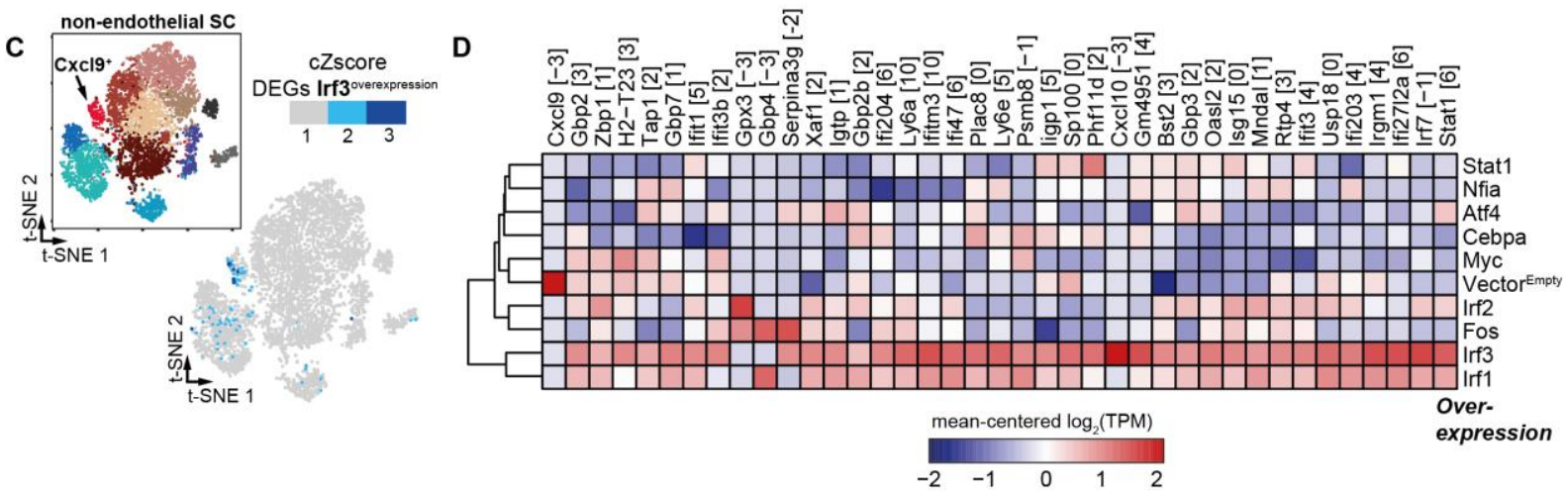

Figure 6. Irf3 promotes the differentiation of mesenchymal stem cells towards a $\mathrm{Cxcl9}^{+} \mathrm{FRC}_{\text {molecular }}$ phenotype. Murine $\mathrm{C} 3 \mathrm{H} 10 \mathrm{~T} 1 / 2$ were lentivirally transduced and puromycin-selected for stable vector integration. TF expression was doxycycline-induced and maintained for 12 days. Subsequently, BRB-seq was performed on the induced cells. (A) Representative phase contrast images of morphological phenotypes subsequent to overexpression-driven differentiation (scale bar $=30 \mu \mathrm{m}$ ). (B) The heatmap depicts the cumulative Z-score (cZscore) of DEGs, compared to Vector ${ }^{\text {Empty }}$, at "TF overexpression" in the C3H10T1/2 cell line among the Top 100 DEGs per LN subset (Supp. Table S4). The percentage indicates the proportion of DEGs that were identified per subset (see Figure 3, Supp. Table S4) and the DEGs identified with "TF overexpression" in C3H10T1/2. (C) The cumulative Z-score on a per cell basis of the significant DEGs due to Irf3 overexpression superimposed on the scRNA-seq developmental map. (D) The heatmap depicts all detected DEGs from the Cxc19+ cluster for the tested (overexpressed) TFs. cZscore, cumulative Z-score; DEG, differentially expressed gene; TF, transcription factor; FRC, reticular fibroblastic stromal cell; SC, stromal cell; TPM, transcripts per kilobase of exon per million reads. 


\section{Discussion}

In our study, we set out to map the epigenomic landscape of the non-endothelial SC compartment of both skin- and gut-draining LNs. Together with scRNA-seq profiling along postnatal development, we increased our understanding of four key aspects of the LNs nonendothelial SC compartment: 1) LNSCs are marked by a tissue-specific, microbiotaindependent epigenomic landscape; 2) within pLN SCs, defined TFs propel transcriptional upregulation of pro-inflammatory cytokines; 3) Postnatal segregation of mesenchymal progenitors is driving the expansion of the developing $\mathrm{LN}$; and 4) $\mathrm{Cxcl}^{+} \mathrm{FRC}$ differentiation is driven predominantly by $\operatorname{Irf} 3$.

LNSCs have long been recognized as key structural organizers, but are also increasingly perceived as effective modulators of immune responses, ${ }^{8,23}$ even in a location-specific manner. ${ }^{11,21,22}$ However, whether tissue-specific immuno-modulatory properties of the nonendothelial SC compartment are retained in the epigenomic landscape is so far understudied. ${ }^{25}$ Importantly, when we compared both chromatin accessibility and DNA methylation using ATAC-seq and WGBS, respectively, we observed striking differences between skin- and gutdraining LNs, yet we could exclude a key contribution by microbial colonization. Although several studies have shown that the microbiota can impinge on the epigenome of the host's adaptive immune system, ${ }^{51}$ its influence does not seem to extend to the draining LNs SCs. This finding was surprising, as we had previously demonstrated that mLNs from GF mice lose their high Treg-inducing capacity upon transplantation into a skin-draining site, while the mLNs from SPF-housed mice persistently maintained their immuno-modulatory functions. ${ }^{11,22}$ Hence, the precise epigenomic modifications and the involved SC subsets that enable microbiotadependent imprinting of the differential Treg-inducing capacity remain to be identified, a feat that should be attainable, considering the increasing sensitivity of methods to profile histone modifications and TF binding, ${ }^{52,53}$ that align with the low cellular yields of ex vivo SCs. We would like to point out that the currently applied sorting of $\mathrm{CD}^{-} 5^{-\mathrm{CD}} 31^{-} \mathrm{gp} 38^{+}$cells, also applied for the epigenomic profiling in the presented study, yields a heterogeneous cell population that could very well mask epigenomic modifications on a per subset basis. Hence, future studies should consider to at least distinguish between the two dominant SC types in the mLN, namely FRCs and CD $34^{+}$SCs.

Previous studies underlined the tissue-specific immuno-modulatory functions of LNSCs, with the skin-draining pLN SCs tending to reside in a more pro-inflammatory state as compared to mLN SCs. ${ }^{11,34,54}$ We thus aimed to delineate epigenomic modifications that maintain the immuno-modulatory function of LNSCs. By performing motif enrichment analysis together with gene expression profiling, we delineated steady-state expression signatures poising $\mathrm{pLN}$ SCs for pro-inflammatory responses and identified TF modules that likely regulate the expression of dormant accessible genes in mLN SCs. The higher expression of proinflammatory mediators aligns with previous studies, ${ }^{11,34}$ but the observations here point towards an active TF-based regulation of pro-inflammatory responses in pLN SCs that relies on chromatin features that are equally accessible across gut- and skin-draining LNSCs. The distinctly higher expression of several interferon regulatory factors, including Irfl, Irf5 and Irf8 under steady-state in pLN SCs, thus enables the skin-draining LNs to rapidly respond to infectious incursions. Together with the elevated steady-state expression of pro-inflammatory chemotactic mediators, this enforces the propensity of the pLN SC compartment to support proinflammatory responses; an epigenetically imprinted feature not maintained within the mLN SCs.

We initially set out to map postnatal SC development to identify TFs that drive the differentiation of distinct subsets and correspond to the epigenomic TF footprint. scRNA-seq 
profiling along postnatal development identified two distinct subsets of putative proliferating progenitors that harbor the potential to yield either FRCs or $\mathrm{CD} 34^{+} \mathrm{SCs}$. The surprising observation of distinct postnatal SC subsets with progenitor potential, extends the perception that $\mathrm{Cxcl}_{13} 3^{+}$LTos are the sole subset that contributes to the expanding non-endothelial SC pool. ${ }^{8,23,41,55}$ Although we cannot formally exclude that $\mathrm{CD} 34^{+}$Prog cells originate from Cxcl13 ${ }^{+}$LTos and can not make a detailed statement regarding the precise Cxcl13 ${ }^{+}$LTo and $\mathrm{Cxcl}^{+} 3^{+}$Prog progenitor-progeny relationship, the inferred differentiation trajectories underscored the distinct gene expression profiles between $\mathrm{Cxcl}_{13}{ }^{+}$Prog and $\mathrm{CD} 34^{+}$Prog cells, supporting the notion that these subsets give rise to either FRCs or CD34 ${ }^{+} \mathrm{SCs}$. It is conceivable that $\mathrm{Cxcl}^{+} 3^{+}$Prog cells within mLNs have already established a distinct branch at birth, suggesting that a proliferating subset of commonly identified $\mathrm{Cxcl}_{13}{ }^{+}$LTos is contributing to the postnatal expansion of the $\mathrm{LN}$ by transitioning through the $\mathrm{Cxcl}_{13}{ }^{+}$Prog state. In contrast, $C d 34$ is expressed in three different locations in the neonatal mLN parenchyma: the capsule, the medulla and the adventitia of major vessels. ${ }^{10,12}$ We noted that large vessels surrounded by an adventitial layer are scarce within the postnatal mLN, thus making it unlikely that the majority of the $\mathrm{CD} 34^{+}$cells are of adventitial origin, which is mirrored by the distinct subsetspecific gene expression profiles. However, to truly delineate the contribution of different nonendothelial progenitor pools, a spatio-temporal mapping of developing mLNs at pre- and postnatal stages would be required.

Regardless, the postnatal scRNA-seq profiling already enabled us to identify putative progenitor pools and TFs that contribute to the differentiation of distinct subsets of nonendothelial SC in adult mLNs. The identfication of those TFs enables targeted in vivo and in vitro verification of the determinants that drive the postnatal development of non-endothelial SCs. As none of the canonical markers for distinct subsets of the CD34 ${ }^{+} \mathrm{SCs}$ or FRCs trajectory are uniquely expressed on a per subset basis, utilization of mouse models involving floxed TFs in conjunction with CD34- or Ccl19-Cre would target larger and less fine-grained subsets of the LN SC compartment. We therefore opted for an in vitro TF overexpression model using a MSC line, which enabled us to screen multiple TFs and avoided the requirement to utilize neonatal progenitor cells, which are scarce and can so far not be isolated due to the lack of canonical markers. The majority of the overexpressed TFs did not induce a differentiation towards a phenotypical profile that resembles ex vivo SCs, although a substantial number of TFs supported fibroblastic morphology. While the utilized C3H10T1/2 cell line is pre-disposed towards an adipogenic, osteogenic, myogenic or chondrogenic differentiation, ${ }^{58}$ we did observe that overexpression of TFs from the IRF family induced a fibroblastic phenotype, likely due to the embryonic origin and thus multi-potency of the utilized MSCs. ${ }^{59}$ Particularly striking was the observation that Irf3 drives the differentiation of cells towards a molecular phenotype that resembles $\mathrm{Cxcl}^{+}$FRCs, while Irf1/Irf2 support this specific molecular phenotype to a lesser extent. Within the $\mathrm{LN}$, expression of $\mathrm{Cxcl9}$ by $\mathrm{Cxcl}^{+} \mathrm{FRCs}$ is critically required to establish the chemotactic driving forces that enable the initial detection of pathogenic incursions. ${ }^{20}$ The distinct positioning of the proportionally small cellular compartment of $\mathrm{Cxcl}^{+} \mathrm{FRCs}$ in close proximity to lymphatic entry points enables rapid detection of tissue-originated pathogenic incursions, and subsequently accumulation of memory $\mathrm{CD} 8^{+} \mathrm{T}$ cells. ${ }^{12,20}$ Importantly, $\mathrm{Cxcl}^{+}$FRCs are conserved across different LNs including skin- and gut-draining tissues. ${ }^{11,12}$ Irf3 overexpression established robust upregulation of various interferon response elements, in line with the up-regulation of Stat1, but did not result in an elevated expression of Cxcl9, a process which could rely either on additional stimulators such as IFN $\beta$ or the requirement of combinatorial TF expression. ${ }^{60,61}$ Surprisingly, the $\mathrm{Cxcl}^{+}$FRCs could neither be annotated to the $\mathrm{CD}_{3} 4^{+} \mathrm{SC}$ nor the FRC developmental trajectories and showed varying expression for Ccl19, Ly6cl and Bst1, indicating that $\mathrm{Cxcl}^{+}$FRCs are still heterogeneous themselves. 
We anticipate that spatial- and subset-specific transcriptional and epigenomic profiling applied to non-endothelial SCs will further identify defining features of the immuno-modulatory potential of the heterogeneous non-endothelial SCs of mLNs. Nevertheless, the transcriptomic analysis of the postnatally developing $\mathrm{mLN}$ together with the epigenomic profiling across skinand gut-draining LNs, can function as a guiding resource to dissect LNSC expansion and immuno-modulation; paving the way to identify entry points to tissue-specifically modulate immune responses by leveraging the embedded SC immune memory and unique functional properties. 


\section{References:}

1. Cupedo, T. et al. Presumptive Lymph Node Organizers are Differentially Represented in Developing Mesenteric and Peripheral Nodes. J. Immunol. 173, 2968-2975 (2004).

2. Onder, L. \& Ludewig, B. A Fresh View on Lymph Node Organogenesis. Trends Immunol. (2018). doi:10.1016/j.it.2018.08.003

3. Onder, L. et al. Lymphatic Endothelial Cells Control Initiation of Lymph Node Organogenesis. Immunity (2017). doi:10.1016/j.immuni.2017.05.008

4. Krishnamurty, A. T. \& Turley, S. J. Lymph node stromal cells: cartographers of the immune system. Nature Immunology 21, 369-380 (2020).

5. White, A. et al. Lymphotoxin a-dependent and -independent signals regulate stromal organizer cell homeostasis during lymph node organogenesis. Blood 110, 1950-1959 (2007).

6. Bovay, E. et al. Multiple roles of lymphatic vessels in peripheral lymph node development. J. Exp. Med. 215, 2760-2777 (2018).

7. Cupedo, T. et al. Initiation of Cellular Organization in Lymph Nodes Is Regulated by Non-B Cell-Derived Signals and Is Not Dependent on CXC Chemokine Ligand 13. J. Immunol. 173, 4889-4896 (2004).

8. Chang, J. E. \& Turley, S. J. Stromal infrastructure of the lymph node and coordination of immunity. Trends Immunol 36, 30-39 (2014).

9. Chai, Q. et al. Maturation of lymph node fibroblastic reticular cells from myofibroblastic precursors is critical for antiviral immunity. Immunity 38, 1013-24 (2013).

10. Sitnik, K. M. et al. Context-Dependent Development of Lymphoid Stroma from Adult CD34+ Adventitial Progenitors. Cell Rep. 14, 2375-2388 (2016).

11. Pezoldt, J. et al. Neonatally imprinted stromal cell subsets induce tolerogenic dendritic cells in mesenteric lymph nodes. Nat. Commun. 9, 3903 (2018).

12. Rodda, L. B. et al. Single-Cell RNA Sequencing of Lymph Node Stromal Cells Reveals Niche-Associated Heterogeneity. Immunity 0, (2018).

13. Koning, J. J. et al. Nestin-Expressing Precursors Give Rise to Both Endothelial as well as Nonendothelial Lymph Node Stromal Cells. J. Immunol. 197, 2686-94 (2016).

14. Jarjour, M. et al. Fate mapping reveals origin and dynamics of lymph node follicular dendritic cells. J. Exp. Med. 211, 1109-22 (2014).

15. Gentek, R. \& Bajénoff, M. Lymph Node Stroma Dynamics and Approaches for Their Visualization. Trends Immunol. 38, 236-247 (2017).

16. Cremasco, V. et al. B cell homeostasis and follicle confines are governed by fibroblastic reticular cells. Nat Immunol 15, 973-981 (2014).

17. Onder, L. et al. IL-7-producing stromal cells are critical for lymph node remodeling. Blood (2012). doi:10.1182/blood-2012-03-416859

18. Siegert, S. et al. Fibroblastic reticular cells from lymph nodes attenuate T cell expansion by producing nitric oxide. PLoS One 6, e27618 (2011).

19. Lukacs-Kornek, V. et al. Regulated release of nitric oxide by nonhematopoietic stroma controls expansion of the activated T cell pool in lymph nodes. Nat Immunol 12, 10961104 (2011).

20. Kastenmüller, W. et al. Peripheral Prepositioning and Local CXCL9 ChemokineMediated Guidance Orchestrate Rapid Memory CD8+ T Cell Responses in the Lymph Node. Immunity 38, 502-513 (2013).

21. Molenaar, R. et al. Lymph node stromal cells support dendritic cell-induced guthoming of T cells. J. Immunol. 183, 6395-402 (2009).

22. Cording, S. et al. The intestinal micro-environment imprints stromal cells to promote efficient Treg induction in gut-draining lymph nodes. Mucosal Immunol 7, 359-368 (2014). 
23. Pikor, N. B., Cheng, H.-W., Onder, L. \& Ludewig, B. Development and Immunological Function of Lymph Node Stromal Cells. J. Immunol. 206, 257-263 (2021).

24. Krausgruber, T. et al. Structural cells are key regulators of organ-specific immune responses. Nature 583, 296-302 (2020).

25. Oltz, E. M. \& Schwab, S. Stromal Immunology: Frameworks for Development and Response. J. Immunol. 206, 241-242 (2021).

26. Zou, M., Wiechers, C. \& Huehn, J. Lymph node stromal cell subsets-Emerging specialists for tailored tissue-specific immune responses. Int. J. Med. Microbiol. 311, 151492 (2021).

27. Buechler, M. B. \& Turley, S. J. A short field guide to fibroblast function in immunity. Seminars in Immunology (2018). doi:10.1016/j.smim.2017.11.001

28. Hansen, K. D., Langmead, B. \& Irizarry, R. A. BSmooth: from whole genome bisulfite sequencing reads to differentially methylated regions. Genome Biol. 13, R83 (2012).

29. Hisamatsu, D., Ohno-Oishi, M., Nakamura, S., Mabuchi, Y. \& Naka-Kaneda, H. Growth differentiation factor 6 derived from mesenchymal stem/stromal cells reduces age-related functional deterioration in multiple tissues. Aging (Albany. NY). 8, 1259-75 (2016).

30. Christiansen, A. J. et al. Lymphatic endothelial cells attenuate inflammation via suppression of dendritic cell maturation. Oncotarget 7, 39421-39435 (2016).

31. Katakai, T. et al. Organizer-like reticular stromal cell layer common to adult secondary lymphoid organs. J. Immunol. 181, 6189-200 (2008).

32. Molenaar, R. et al. Expression of retinaldehyde dehydrogenase enzymes in mucosal dendritic cells and gut-draining lymph node stromal cells is controlled by dietary vitamin A. J Immunol 186, 1934-1942 (2011).

33. Zhu, H., Wang, G. \& Qian, J. Transcription factors as readers and effectors of DNA methylation. Nat. Rev. Genet. 17, 551-565 (2016).

34. Malhotra, D. et al. Transcriptional profiling of stroma from inflamed and resting lymph nodes defines immunological hallmarks. Nat Immunol 13, 499-510 (2012).

35. Jia, G. et al. Single cell RNA-seq and ATAC-seq analysis of cardiac progenitor cell transition states and lineage settlement. Nat. Commun. 9, 1-17 (2018).

36. Berg, D. A. et al. A Common Embryonic Origin of Stem Cells Drives Developmental and Adult Neurogenesis. Cell 177, 654-668.e15 (2019).

37. Eckert, N., Permanyer, M., Yu, K., Werth, K. \& Förster, R. Chemokines and other mediators in the development and functional organization of lymph nodes. Immunol. Rev. 289, 62-83 (2019).

38. Grisaru, S., Cano-Gauci, D., Tee, J., Filmus, J. \& Rosenblum, N. D. Glypican-3 Modulates BMP- and FGF-Mediated Effects during Renal Branching Morphogenesis. Dev. Biol. 231, 31-46 (2001).

39. van de Pavert, S. A. \& Mebius, R. E. New insights into the development of lymphoid tissues. Nat. Rev. Immunol. 10, 664-674 (2010).

40. Katakai, T. Marginal reticular cells: a stromal subset directly descended from the lymphoid tissue organizer. Front Immunol 3, 200 (2012).

41. Perez-Shibayama, C., Gil-Cruz, C. \& Ludewig, B. Fibroblastic reticular cells at the nexus of innate and adaptive immune responses. Immunol. Rev. 289, 31-41 (2019).

42. Trapnell, C. et al. The dynamics and regulators of cell fate decisions are revealed by pseudotemporal ordering of single cells. Nat. Biotechnol. 32, 381-6 (2014).

43. Barozzi, I. et al. Coregulation of transcription factor binding and nucleosome occupancy through DNA features of mammalian enhancers. Mol. Cell 54, 844-57 (2014).

44. Iwafuchi-Doi, M. \& Zaret, K. S. Pioneer transcription factors in cell reprogramming. 
Genes Dev. 28, 2679-92 (2014).

45. Stadler, M. B. et al. DNA-binding factors shape the mouse methylome at distal regulatory regions. Nature 480, 490-5 (2011).

46. Huynh-Thu, V. A. \& Geurts, P. dynGENIE3: dynamical GENIE3 for the inference of gene networks from time series expression data. Sci. Rep. 8, 3384 (2018).

47. Gubelmann, C. et al. Identification of the transcription factor ZEB1 as a central component of the adipogenic gene regulatory network. Elife 3, 1-30 (2014).

48. Reznikoff, C. A., Brankow, D. W. \& Heidelberger, C. Establishment and Characterization of a Cloned Line of C3H Mouse Embryo Cells Sensitive to Postconfluence Inhibition of Division. Cancer Res. 33, 3231-3238 (1973).

49. Hiraike, Y. et al. NFIA differentially controls adipogenic and myogenic gene program through distinct pathways to ensure brown and beige adipocyte differentiation. PLOS Genet. 16, e1009044 (2020).

50. Alpern, D. et al. BRB-seq: ultra-affordable high-throughput transcriptomics enabled by bulk RNA barcoding and sequencing. Genome Biol. 20, 71 (2019).

51. Pasztoi, M., Pezoldt, J., Huehn, J. \& Maria Pasztoi Jochen Huehn, J. P. Microenvironment Matters: Unique Conditions Within Gut-Draining Lymph Nodes Favor Efficient De Novo Induction of Regulatory T Cells. Prog. Mol. Biol. Transl. Sci. 136, 35-56 (2015).

52. Ranzoni, A. M. et al. Integrative Single-Cell RNA-Seq and ATAC-Seq Analysis of Human Developmental Hematopoiesis. Cell Stem Cell 28, 472-487.e7 (2021).

53. Grosselin, K. et al. High-throughput single-cell ChIP-seq identifies heterogeneity of chromatin states in breast cancer. Nat. Genet. 51, 1060-1066 (2019).

54. Gregory, J. L. et al. Infection Programs Sustained Lymphoid Stromal Cell Responses and Shapes Lymph Node Remodeling upon Secondary Challenge. Cell Rep. 18, 406418 (2017).

55. Cheng, H.-W. et al. Origin and differentiation trajectories of fibroblastic reticular cells in the splenic white pulp. Nat. Commun. 10, 1739 (2019).

56. Koning, J. J. et al. Development of follicular dendritic cells in lymph nodes depends on retinoic acid mediated signaling. bioRxiv 2020.05.20.106385 (2020).

doi:10.1101/2020.05.20.106385

57. Randall, T. D., Carragher, D. M. \& Rangel-Moreno, J. Development of secondary lymphoid organs. Annual Review of Immunology 26, 627-650 (2008).

58. Tang, Q. Q., Otto, T. C. \& Lane, M. D. Commitment of C3H10T1/2 pluripotent stem cells to the adipocyte lineage. Proc. Natl. Acad. Sci. U. S. A. 101, 9607-9611 (2004).

59. Zhao, L., Li, G., Chan, K. M., Wang, Y. \& Tang, P. F. Comparison of multipotent differentiation potentials of murine primary bone marrow stromal cells and mesenchymal stem cell line C3H10T1/2. Calcif. Tissue Int. 84, 56-64 (2009).

60. Forero, A. et al. Differential Activation of the Transcription Factor IRF1 Underlies the Distinct Immune Responses Elicited by Type I and Type III Interferons. Immunity 51, 451-464.e6 (2019).

61. Parekh, U. et al. Mapping Cellular Reprogramming via Pooled Overexpression Screens with Paired Fitness and Single-Cell RNA-Sequencing Readout. Cell Syst. 7, 548555.e8 (2018).

62. Buenrostro, J. D., Wu, B., Chang, H. Y. \& Greenleaf, W. J. ATAC-seq: A Method for Assaying Chromatin Accessibility Genome-Wide. Curr. Protoc. Mol. Biol. 109, 21.29.1-9 (2015).

63. Picelli, S. et al. Tn5 transposase and tagmentation procedures for massively scaled sequencing projects. Genome Res. 24, 2033-2040 (2014).

64. Andrews, S. FastQC: A quality control tool for high throughput sequence data. http://www.bioinformatics.babraham.ac.uk/projects/fastqc/ 
http://www.bioinformatics.babraham.ac.uk/projects/ (2010). doi:citeulike-articleid: 11583827

65. Martin, M. Cutadapt removes adapter sequences from high-throughput sequencing reads. EMBnet.journal 17, 10 (2011).

66. Dobin, A. \& Gingeras, T. R. Mapping RNA-seq Reads with STAR. Curr. Protoc. Bioinforma. 51, 11.14.1-19 (2015).

67. Zhang, Y. et al. Model-based analysis of ChIP-Seq (MACS). Genome Biol. 9, R137 (2008).

68. Yue, F. et al. A comparative encyclopedia of DNA elements in the mouse genome. Nature 515, 355-364 (2014).

69. Love, M. I., Huber, W. \& Anders, S. Moderated estimation of fold change and dispersion for RNA-seq data with DESeq2. Genome Biology 15, (Cold Spring Harbor Labs Journals, 2014).

70. Zhu, L. J. et al. ChIPpeakAnno: A Bioconductor package to annotate ChIP-seq and ChIP-chip data. BMC Bioinformatics 11, 237 (2010).

71. Heinz, S. et al. Simple Combinations of Lineage-Determining Transcription Factors Prime cis-Regulatory Elements Required for Macrophage and B Cell Identities. Mol. Cell 38, 576-589 (2010).

72. Kim, D. et al. TopHat2: accurate alignment of transcriptomes in the presence of insertions, deletions and gene fusions. Genome Biol. 14, R36 (2013).

73. Anders, S., Pyl, P. T. \& Huber, W. HTSeq-A Python framework to work with highthroughput sequencing data. Bioinformatics 31, 166-169 (2015).

74. Alexa, A. \& Rahnenfuhrer, J. topGO: topGO: Enrichment analysis for Gene Ontology. R package version 2.18.0. October (2010).

75. Shannon, P. et al. Cytoscape: A software Environment for integrated models of biomolecular interaction networks. Genome Res. 13, 2498-2504 (2003). 


\section{Methods}

\section{Mice}

CD90.1 mice (BALB/c) were bred and kept under SPF conditions in isolated ventilated cages at the Helmholtz Centre for Infection Research (Braunschweig, Germany). GF mice (BALB/c) were generated at Hannover Medical School (Hannover, Germany) by cesarean section and maintained either in plastic film isolators or in static micro-isolators (gnotocages) at Hannover Medical School or the Helmholtz Centre for Infection Research (Braunschweig, Germany). If not stated otherwise, water and food were supplied ad libitum. In all experiments, gender- and age-matched mice were used. All mice were housed and handled in accordance with good animal practice as defined by FELASA and the national animal welfare body GV-SOLAS.

\section{Antibodies}

Fluorochrom-conjugated anti-human anti-CD24 (clone M1/69, APC, BioLegend Cat. \#101814), anti-CD31 (clone 390, PE-Cy7, BioLegend Cat. \#102418), anti-CD45 (clone 30-F11, APC, BioLegend Cat. \#103112), anti-gp38 (clone 8.1.1, PE, BioLegend Cat. \#127408) and Ter119 (clone Ly-76, APC, Biolegend Cat. \#116212) were utilized in this study.

\section{RNAscope FISH}

Triple hybridizations were carried out using the RNAscope Multiplex Fluorescent Detection Kit v2 (Advanced Cell Diagnostics, Cat. \#320871 \#300041 \#323100 \#310023 \#310018) in combination with the corresponding 4-Plex Ancillary Kit (Advanced Cell Diagnostics, Cat \#323120 \#321831). The following target probes were used: Mm-Cdk1 (Cat. \#476081, targeting bp 58-1159), Mm-Cxcl13 (Cat. \#406311-C2, targeting bp 2-1143), Mm-Cc119 (Cat. \#432881-C2, targeting bp 5-712), Mm-CD34 (Cat. \#319161-C3, targeting bp 383-1590) and Mm-CD31(Pecam1) (Cat. \#316721-C4, targeting bp 915-1827). Sample preparation and stainings were carried out according to manufacturer's instructions. In brief, mLNs from 0-1d old SPF-housed mice were dissected and fixed in $10 \%$ neutrally buffered formaldehyde for 16$32 \mathrm{~h}$ at RT, washed with 1x PBS (Gibco, Cat. \#14190169), and dehydrated in a series of ethanol and xylene submersions before embedding in paraffin (Merck, Cat. \#76242). Formalin-fixed paraffin-embedded (FFPE) tissue blocks were stored at $4^{\circ} \mathrm{C}$. Sliced $3 \mu \mathrm{m}$ tissue sections were continuously stored at $4^{\circ} \mathrm{C}$ until RNAscope stainings were performed. FFPE sections were baked at $60^{\circ} \mathrm{C}$ for $1 \mathrm{~h}$, before being deparaffinized and dehydrated. Tissue sections were incubated with hydrogen peroxide for $10 \mathrm{~min}$ at RT before target retrieval was carried out in a steamer (Braun, Type 3216) for $15 \mathrm{~min}$ at $>98^{\circ} \mathrm{C}$. Protease treatment was performed with Protease Plus (Advanced Cell Diagnostics, Cat. \#322380) for $20 \mathrm{~min}$ in a humidified hybridization chamber at $40^{\circ} \mathrm{C}$. Subsequently, probes were allowed to hybridize to their targets 
for $2 \mathrm{~h}$ at $40^{\circ} \mathrm{C}$ in the hybridization chamber. During the following horse radish peroxidase (HRP) based amplification process, the tyramide signal amplification (TSA)-conjugated fluorophores Opal520, Opal570 and Opal650 (Perkin Elmer, Cat. \#NEL80001KT) were used to visualize target probes. Tissue sections were counterstained with DAPI (Merck, Cat. \#D9542) and mounted with ProLong Gold Antifade Mountant (Thermo Fisher Scientific, Cat. \#P10144). Sections incubated with negative control probes (DapB) were stained in parallel and a mix of positive control probes (POLR2A, PPIB, UBC, Hprt) was utilized to confirm RNA integrity in each assessed tissue block. Images were acquired with an Olympus VS120 slide scanner fluorescence microscope using the VS-ASW-FL software (Olympus). Z-stacks were acquired at $40 \mathrm{x}$ or $20 \mathrm{x}$ magnification and extended focus imaging (EFI) was done at 20x magnification.

\section{Stromal cell isolation}

For SC isolation, skin-draining pLNs (inguinal and axillary or popliteal) or mLNs (small intestinal and colon/caecum-draining) were resected and digested in RPMI 1640 medium (Gibco, Cat. \#72400021) containing $0.2 \mathrm{mg} / \mathrm{ml}$ collagenase P (Roche, Cat. \#11213865001), $0.15 \mathrm{U} / \mathrm{ml}$ dispase (Roche, Cat. \#4942078001) and $0.2 \mathrm{mg} / \mathrm{ml}$ DNase I (Roche, Cat. \#4536282001) as described previously. ${ }^{34}$ After digestion, cells were kept at $4{ }^{\circ} \mathrm{C}$ in $\mathrm{PBS}$ containing 0.2 \% BSA (Merck, Cat. \#A2058) and 5 mM EDTA (Roth, Cat. \#8043.1). CD45cells were enriched by autoMACS separation after magnetic labeling of $\mathrm{CD} 45^{+}$cells using anti-CD45-APC followed by anti-APC microbeads (Miltenyi Biotec, Cat. \#130-090-855) or anti-CD45 Nanobeads (Biolegend, Cat. \#480028). Subsequently, the CD45- fraction was stained using fluorochrome-coupled antibodies and used to sort CD45-CD24-CD31 ${ }^{-} \mathrm{gp} 38^{+} \mathrm{FSCs}$ (Aria II, $100 \mu \mathrm{m}$ nozzle) and bulk CD45-CD24- non-hematopoietic cells (Aria III, $70 \mu \mathrm{m}$ nozzle) for RNA-seq, ATAC-seq and scRNA-seq.

\section{Transfection and lentiviral packing}

Reverse lentiviral transfection was performed using Lipofectamine 2000 (Thermo Fisher, Cat. \#11668027) following the manufacturer's instruction. TF-bearing lentiviral vectors ${ }^{47}$ and lentiviral packaging plasmids pRSV-Rev (addgeneID 12253), pMDLg/pRRE (addgeneID 12251) and pCMV-VSV-G (addgeneID 8454) were supplemented at the ratio 1:1:1:1 and incubated for $30 \mathrm{~min}$ at the room temperature. Prior to transfection, HEK 293T cells (ATCC Cat. No. SD-3515) were washed with PBS (Thermo Fisher, Cat. \#14190169) and dissociated using 0.25\% Trypsin-EDTA (Life Technologies, Cat. \#25200056). Cells were resuspended in DMEM (Gibco, 41966029) 10\% FBS (Gibco, Cat. \#10270106) and 1\% penicillin-streptomycin (Life Technologies, Cat. \#15140-122) and seeded in individual wells at confluence of 95\% with 
the transfection mix. $12 \mathrm{~h}$ post-transfection, fresh medium was added to attached cells and the virus-containing supernatant collected after $48 \mathrm{~h}$, dead cells removed by centrifuging at $300 \mathrm{~g}$ for 10 min and supernatant stored at $-80^{\circ} \mathrm{C}$ for up to 6 months.

\section{Lentiviral transduction}

Murine C3H10T1/2 cells (ATCC Cat. No. CCL-226) were seeded $12 \mathrm{~h}$ prior to transduction at confluence of 10-20\%. Transfection lentivirus-containing supernatant was mixed at 1:1 ratio with fresh medium and $10 \mu \mathrm{l} / \mathrm{ml}$ polybrene (Sigma, Cat. \#TR-1003-G) and added to the plated adherent C3H10T1/2 cells. Cells were then centrifuged at $1300 \mathrm{~g}$ for $30 \mathrm{~min}$ at $37^{\circ} \mathrm{C}$, incubated with the respective lentivirus for $24 \mathrm{~h}$ and fresh medium was added after $24 \mathrm{~h}$. After $48 \mathrm{~h}$, transduced cells were selected using $2 \mu \mathrm{l} / \mathrm{ml}$ of puromycin (Thermo Fisher, Cat. \#A1113803) for $72 \mathrm{~h}$. Subsequent to puromycin selection, medium was replaced and puromycin-resistant cells permitted to recover for $48 \mathrm{~h}$. Then, medium containing $2 \mu \mathrm{l} / \mathrm{ml}$ doxycycline was added (Sigma, Cat. \#D9891-1G) to induce TF expression. Medium with doxycycline was replenished every $48 \mathrm{~h}$ and doxocycline treatment maintained for 12 days. Direct-zol RNA kit (Zymo Research, Cat. \#R2052) was used to extract RNA according to the manufacturer's instruction.

\section{Library preparation WGBS}

Genomic DNA was isolated from purified CD45CD31-Ter119gp38 ${ }^{+}$using the AllPrep DNA/RNA Micro Kit (Qiagen, Cat. \#80284) according to the manufacturer's instructions. Concentration and quality of the purified genomic DNA (gDNA) was determined by using NanoDrop (Thermo Fisher Scientific). Fragmentation of gDNA was carried out via Covaris S2 (Covaris), at duty cycle 10\%, intensity 4 and 200 cycles per burst during $80 \mathrm{sec}$, to obtain fragments with an average length of $300 \mathrm{bp}$. The size of the fragments was verified with Agilent Technologies 2100 Bioanalyzer. DNA sequencing libraries were generated from fragmented gDNA using the TruSeq DNA Sample Prep Kit v2 (Illumina, Cat. \#15026486) according to the manufacturer's instructions and extending the workflow by adding one additional step: Subsequent to ligation of the adapter molecules to the DNA fragments, the sample was subjected to bisulfite conversion reaction using the EZ DNA Methylation Kit (Zymo Research, Cat. \#D5001). The protocol for the True Seq DNA generation was then followed. The bisulfiteconverted library was amplified by performing a PCR reaction $\left(10\right.$ cycles, $98^{\circ} \mathrm{C}$ for $10 \mathrm{~s}, 63^{\circ} \mathrm{C}$ for $30 \mathrm{~s}, 72^{\circ} \mathrm{C}$ for $1 \mathrm{~min}$ ) including the TruSeq primer mix and the KAPA Hifi Uracil+ Polymerase Master Mix (Kapa Biosystems, Cat. \#KK2801). The PCR product was purified and size controlled by Agilent Technologies 2100 Bioanalyzer (High Sensitivity DNA Chip). The libraries were sequenced on an Illumina HiSeq2500 sequencer using the TruSeq SBS Kit v3HS (200 cycles, paired end run) with an average of $2 * 10^{8}$ reads per replicate. The WGBS raw 
and processed data are available at NCBI GEO (GSE172526).

\section{Library preparation ATAC-seq}

$\mathrm{CD}^{2} 5^{-} \mathrm{CD} 24^{-\mathrm{CD}} 31^{-} \mathrm{gp} 38^{+}$cells were sorted by FACS into PBS containing 0.2\% BSA. Cells were washed once with PBS before DNA transposition was performed with the Nextera DNA Library Prep Kit (Illumina, Cat. \#FC-121-1031). Per sample, $25 \mu 1$ TD, $2.5 \mu 1$ TDE1 and $22 \mu 1$ nuclease-free water were combined and placed at $37^{\circ} \mathrm{C}$ for $3 \min$ before $0.5 \mu 1$ of $1 \%$ Digitonin (Promega, Cat. \#G9441) was added to the master mix. Samples were resuspended in the transposition reaction mix and incubated for $30 \mathrm{~min}$ at $37^{\circ} \mathrm{C}$ at $300 \mathrm{rpm}$. After transposition, DNA was purified with the MinElute PCR Purification kit (Qiagen, Cat. \#28006) according to manufacturer's instructions and eluted in $50 \mu \mathrm{l}$ nuclease free water. Transposed DNA fragments were pre-amplified using $10 \mu \mathrm{l}$ transposed DNA, $10 \mu \mathrm{l}$ nuclease free water, $2.5 \mu \mathrm{l}$ $25 \mu \mathrm{M}$ custom Nextera PCR primer 1, $2.5 \mu 125 \mu \mathrm{M}$ custom Nextera PCR primer 2, $25 \mu 1$ NEBNext High-Fidelity 2x PCR Master Mix (New England BioLabs, Cat. \#M0541L) per reaction and amplified via a 6-cycle PCR program $\left(1\right.$ cycle of $72^{\circ} \mathrm{C}$ for $5 \mathrm{~min}, 98^{\circ} \mathrm{C}$ for $30 \mathrm{~s}$; 5 cycles of $98^{\circ} \mathrm{C}$ for $10 \mathrm{~s}, 63^{\circ} \mathrm{C}$ for $30 \mathrm{~s}, 72^{\circ} \mathrm{C}$ for $1 \mathrm{~min}$ ). The forward primer was identical for all samples 5'-AATGATACG GCGACCACCGA GATCTACACTC GTCGGCAGCGT CAGATGTG-3', whereas the reverse primer contained distinct barcodes (example underlined) used for demultiplexing 5'-CAAGCAGAAGA CGGCATACGAG ATTCGCCTTA GTCTCGTGGGC TCGGAGATGT-3' ${ }^{62}$. The appropriate amount of further amplification cycles was determined by qPCR using $5 \mu 1$ of the pre-amplified product. Final amplification was carried out with $45 \mu \mathrm{l}$ of previously PCR amplified DNA, $39.7 \mu$ l nuclease free water, $2.25 \mu \mathrm{l} 25 \mu \mathrm{M}$ customized Nextera PCR primer 1, $2.25 \mu \mathrm{l} 25 \mu \mathrm{M}$ customized Nextera PCR Primer 2, $0.81 \mu 1$ 100x SYBR Green I and $45 \mu 1$ NEBNext High-Fidelity 2x PCR Master Mix with the PCR program of 1 cycle of $98^{\circ} \mathrm{C}$ for $30 \mathrm{~s} ; 8-10$ cycles (depending on qPCR results) of $98^{\circ} \mathrm{C}$ for $10 \mathrm{~s}, 63^{\circ} \mathrm{C}$ for $30 \mathrm{~s}, 72^{\circ} \mathrm{C}$ for $1 \mathrm{~min}$. PCR purification was carried out using the MinElute PCR Purification kit. Finally, size selection was performed with SPRIselect beads (Beckmann-Coulter, Cat. \#B23317) with 1.2x for left-side and 0.55x for right-side selection according to manufacturer's instructions. DNA quality, content and fragment size was assessed with Agilent Technologies 2100 Bioanalyzer profiles and Qubit measurements. Libraries were sequenced on an Illumina NovaSeq6000 sequencer using 50 bp single-end reads, and quality of sequenced libraries was verified with FastQC. The ATAC-seq raw and processed data are available at NCBI GEO (GSE172526).

\section{Library preparation RNA-seq}


Total RNA was extracted from FACS-sorted CD45 ${ }^{-} \mathrm{CD} 24^{-} \mathrm{CD} 31^{-} \mathrm{gp} 38^{+}$using the RNeasy Plus Micro Kit (Qiagen, Cat. \#74034). cDNA was synthesized and amplified using template switching technology of the SMART-Seq v4 Ultra Low Input RNA Kit (Clontech Laboratories, Cat. \#R400752), followed by purification using the Agencourt AMPure XP Kit (Beckman Coulter, Cat. \#A63880). Library preparation was performed with Nextera XT DNA Library Prep Kit (Illumina). The Agilent Technologies 2100 Bioanalyzer was used to control quality and integrity of nucleic acids after each step. Deep sequencing was carried out on an Illumina HiSeq2500 sequencer using 50 bp single reads. Sequenced libraries were assessed for read quality using the FastQC tool. The RNA-seq raw and processed data are available at NCBI GEO (GSE172526).

\section{Library preparation scRNA-seq}

Single CD45 ${ }^{-}$CD24 cells were sorted by FACS ARIA III (BD) and collected in PBS containing $0.04 \% \mathrm{w} / \mathrm{v}$ BSA at a density of 400 cells $/ \mu 1$. Chromium ${ }^{\mathrm{TM}}$ Controller was used for partitioning single cells into nanoliter-scale Gel Bead-In-EMulsions (GEMs) and Single Cell 3' reagent kit v2 for reverse transcription, cDNA amplification and library construction (10xGenomics, Cat. \#120236). The detailed protocol was provided by 10xGenomics. SimpliAmp Thermal Cycler was used for amplification and incubation steps (Applied Biosystems). Libraries were quantified by Qubit ${ }^{\mathrm{TM}}$ 3.0 Fluometer (ThermoFisher) and quality checked using 2100 Bioanalyzer with High Sensitivity DNA kit (Agilent). Sequencing was performed in paired-end mode ( 2 x 75 cycles) on an Illumina NextSeq 500 sequencer to attain approximately 75,000 \pm 25,000 reads per single cell. The scRNA-seq raw and processed data are available at NCBI GEO (GSE172526 and GSE106489 for D56 mLN-SPF ${ }^{11}$ ).

\section{Library preparation BRB-seq}

3'end bulk mRNA cDNA library preparation and sequencing was performed following the BRB-seq strategy as previously described. ${ }^{50}$ In brief, $20 \mathrm{ng}$ of total RNA isolated from TF overexpressions group, each represented with four to five replicates of two independent experiments, were reverse transcribed using SuperScriptTM II Reverse Transcriptase (Lifetech, Cat. Cat. \#18064014) with individual barcoded oligo-dT primers, featuring a 12-nt-long sample barcode (IDT). Double-stranded cDNA was generated by second strand synthesis via the nick translation method. To that end, a mix containing $2 \mu$ l of RNAse H (NEB, Cat. \#M0297S), $1 \mu 1$ of E. coli DNA ligase (NEB, Cat. \#M0205 L), $5 \mu$ l of E. coli DNA Polymerase (NEB, Cat. \#M0209 L), $1 \mu \mathrm{l}$ of 10mM dNTP (Thermo Fisher Scientific, Cat. \#0181), $10 \mu \mathrm{l}$ of 5x Second Strand Buffer (100 mM Tris, pH 6.9, [AppliChem, Cat. \#A3452], 25 mM MgCl 2 [Sigma, Cat. \#M2670], 450 mM KCl [AppliChem, Cat. \#A293], 0.8 mM $\beta$-NAD [Sigma, Cat. N1511], 
$60 \mathrm{mM}\left(\mathrm{NH}_{4}\right)_{2} \mathrm{SO}_{4}$ [Fisher Scientific, Cat. \#AC20587]), and $11 \mu \mathrm{l}$ of water was added to $20 \mu 1$ of ExoI-treated first-strand reaction on ice. The reaction was incubated at $16^{\circ} \mathrm{C}$ for $2.5 \mathrm{~h}$. Fulllength double-stranded cDNA was purified with $30 \mu \mathrm{l}(0.6 \mathrm{x})$ of AMPure XP magnetic beads (Beckman Coulter, Cat. \#A63881) and eluted in $20 \mu \mathrm{l}$ of water. cDNA concentration was measured using Qubit, and cDNA quality was assessed using a Fragment Analyzer (Agilent). cDNA was tagmented with in-house Tn5 ${ }^{63}$, and libraries were purified using AMPure XP magnetic beads $(0.6 \mathrm{X})$. The resulting libraries were profiled with a High Sensitivity NGS Fragment Analysis Kit (Advanced Analytical, Cat. \#DNF-474) and measured with the Qubit dsDNA HS Assay Kit (Invitrogen, Cat. \#Q32851) prior to pooling and sequencing on an Illumina NextSeq 500 sequencer utilizing a BRB-seq custom primer and the High Output v2 kit (75 cycles) (Illumina, Cat. \#FC-404-2005). The sequencing configuration is as follows: Read1 21cycles / index i7 8cycles / Read2 55c. The BRB-seq raw and processed data are available at NCBI GEO (GSE172526).

\section{WGBS analysis}

The sequenced $2 \times 100$ bp paired-end libraries of bisulfite-treated reads were quality assessed and trimmed with the FastQC (version 0.11.1) ) $^{64}$ and Cutadapt (version 1.4$)^{65}$ tools. Trimmed libraries were aligned with the bisulfite short read mapping program BSMAP (version 2.4.3) (Xi \& Li, 2009) versus the mouse reference genome (assembly: GRCm38), and the methylation status of each CpG site was called. Methylation profiles of $\mathrm{CpGs}$ with a minimum coverage of five mapped reads in at least two replicates of one condition and significant (based on t-statistics) change in their methylation status, served as input for the detection. Differentially methylated regions (DMRs) were identified using bsmooth with default settings. ${ }^{28}$

\section{ATAC-seq analysis}

Sequencing reads were mapped to mouse genome (mm10) using STAR (version 2.5.3a) ${ }^{66}$ with parameters --runMode alignReads --outSAMtype BAM SortedByCoordinate -outFilterMultimapNmax 1 (assembly: GRCm38). Duplicates were removed using picard MarkDuplicates (https://broadinstitute.github.io/picard/). Peaks were called on de-duplicated bamfiles using macs 2 callpeak with the parameters --broad $-g \mathrm{~mm}-q 0.05$ for each replicate. ${ }^{67}$ Heatmaps of fragment distribution around the TSS were computed using computeMatrix with the referencepoint $-a 3000-b 3000$ and plotted using plotHeatmap. The regions identified via macs 2 were merged across all replicates into one set of regions, by combining peaks over-lapping with at least one base-pair and removing peaks that overlapped with blacklisted regions. ${ }^{68}$ Differential accessibility of raw ATAC-seq counts for each region/peak across all replicates of all samples were normalized across replicates with size factors computed with DESeq2 (version 1.22). ${ }^{69}$ Pairwise comparisons were performed with DESeq2 and differentially accessible regions (DARs) were 
called with an FDR adjusted p-value of less than 0.05 and a fold change (FC) of at least 2. Genomic features were identified via getAnnotation from ChIPpeakANNO. ${ }^{70}$ The cumulative FC of all DARs for one respective gene is represented as the mean of all the FC of all respective DARs. Transcription factor motif enrichment was computed using homer (version 4.9). ${ }^{71}$ Graphics were generated in R using pheatmap and ggplot 2 .

\section{RNA-seq analysis}

Libraries were aligned versus the mouse reference genome assembly GRCm38 using the splice junction mapper Tophat2 v1.2.0 with default parameterization. ${ }^{72}$ Reads aligned to annotated genes were quantified with the HTSeq (version 0.12.4) ${ }^{73}$ and determined read counts served as input to DESeq $2^{69}$ for pairwise detection and quantification of differential gene expression. RPKM (reads per kilobase of exon length per million mapped reads) values were computed for each library from the raw read counts. For scatterplots and heatmaps only genes with an annotated official Gene Symbol were included. Gene ontology (GO) analyses were performed using the R package TopGo. ${ }^{74}$ The $\mathrm{R}$ packages pheatmap and ggplot 2 were used to generate heatmaps or scatterplots, respectively.

\section{scRNA-seq analysis}

Data were processed using Cell Ranger software (version 2.0.0) Count matrices were further processed with Seurat (version 2.3.3). All cells received an identifier which was used as common meta-data throughout the analysis including differentiation trajectories and dynamic gene regulatory networks (see below).

All cells with less than 1,000 or more than 4,600 detected genes per cell were filtered out. Moreover, cells with more than $4.5 \%$ read mapping to mitochondrial genes were removed yielding 15,659 cells passing QC. After filtering, data were default normalized and the 2,000 most variable genes identified. The expression levels of these genes were scaled before performing PCA. The following covariates were regressed out: number of UMIs, percent of mitochondrial reads, percent of ribosomal reads and scores for the proliferation S.Score and G2M.Score computed with CellCycleScoring(). t-SNE dimensionality reduction was performed using the first 12 dimensions of the PCA and resolution set to 1.1. Only clusters (nonendothelial SC) with normalized expression for Pecam < 1 were used for the further analysis amounting to 9,323 cells which were re-embedded as described above (resolution $=1.0$ ). The perivascular mesenchymal cell (PvMC) cluster and clusters classified as adjacent cells were excluded and the remainder cells, numbering 5,658 mesenchymal cells were re-embedded as described above (resolution $=1.0$ ). For subsetting the Prog. subset, 259 cells were re-embedded as described above (resolution $=0.5$, dimsuse $=10$ ). GO analysis was performed for differentially upregulated genes per cluster using TopGO. ${ }^{74}$ 
Differentiation trajectories were analyzed using Monocle (version 2.18.0). ${ }^{42}$ Unsupervised ordering was performed on the 5,658 mesenchymal cells using Monocle2 's DDRTree algorithm based on genes with a mean expression $>0.1$. The trajectory containing all mesenchymal cells, was split based on marker gene expression Vcaml and Cd34 and the annotation of cells belonging to the Prog ${ }^{\mathrm{Cxcl} 13+}$ or $\operatorname{Prog}^{\mathrm{CD} 34+}$ subset to distinct terminal branches. Two separate unsupervised orderings were performed as described above and are referred to as $\mathrm{CD}^{+} 4^{+} \mathrm{SC}$ or FRC trajectory.

Gene regulatory networks were inferred with the dynGENIE3 algorithm, ${ }^{46}$ where the input expression values were based on ordering the genes according to physiological age per branch for each of the two trajectories being CD $34^{+} \mathrm{SC}$ or FRC. The list of candidate TFs was derived from TFBS from ATAC-seq profiling or DMRs within the proximity of TSSs. For network visualization with Cytoscape (version 3.6.0), ${ }^{75}$ only the top 500 links ranked by weight assigned by dynGENIE3 were used. Node centrality and betweenness were calculated with the degree and betweenness functions from the igraph (version 1.2.2) package.

\section{BRB-seq analysis}

After sequencing and standard Illumina library demultiplexing, the fastq-files were aligned to the mouse reference genome mm10 (GRCm38) using STAR (version 2.7.3a), excluding multiple mapped reads. Resulting BAM files were demultiplexed per sample using BRBseqTools (version 1.4, https://github.com/DeplanckeLab/BRB-seqTools) and read-count matrices generated using HTSeq (version 0.12.4). Raw read counts were converted to transcripts per kilobase of exon per million reads values. Protein-coding genes with at least 5 reads in at least two replicates were included in the analysis. The calculated read counts were further processed with $D E S e q 2$ for quantification of differential gene expression. ${ }^{69}$ Genes were considered as differentially expressed at fold change $>2.0$ and the FDR adjusted p-value of $<0.05$.

\section{Statistical analysis}

For all scripts written in R, we used version 3.4.1 unless otherwise noted.

\section{Data Availability}

ATAC-seq, WGBS, RNA-seq, scRNA-seq and BRB-seq raw and processed data generated during this study are available at NCBI GEO (GSE172526).

\section{Code Availability}

Code used for this study will be made available upon request. 


\section{Acknowledgements}

We thank the Cell Sorting facility (HZI) for cell sorting, the Genome Analytics facility (HZI) for RNA-seq, scRNA-seq, WGBS and ATAC-seq sequencing, and the Genome Analytics facility (EPFL-SV) for BRB-seq sequencing. We thank the Department of Experimental Immunology and especially Dr. Lothar Gröbe and Maria Höxter for their technical assistance. This work was supported by the Hannover Biomedical Research School (HBRS), the Center for Infection Biology (ZIB) from Hannover Medical School, as well as the Deutsche Forschungsgemeinschaft (DFG, German Research Foundation) under Germany's Excellence Strategy - EXC 2155 "RESIST” - Project ID 39087428, SPP1656 (Ho2236/9-2), and PE 2840/1-1). P.A. was supported by the Interdisciplinary Center for Clinical Research (IZKF) of the University Hospital of Wuerzburg (Project Z-6). The Helmholtz Institute for RNA-based Infection Research (HIRI) supported this work with a seed grant through funds from the Bavarian Ministry of Economic Affairs and Media, Energy and Technology (Grant allocation nos. 0703/68674/5/2017 and 0703/89374/3/2017).

\section{Author contributions}

J.P., C.W., M.Bi., M.Z., W.C., S.F., M.E., J.R. and P.A. performed experiments and interpreted data. J.P., M.L., V.G., M.Be., C.W. and E.V. performed bioinformatics analysis. B.D., K.S., A.-E.S. and W.C. provided expertise and feedback. J.P., C.W., and J.H. designed research, interpreted data, and wrote the manuscript.

\section{Competing financial interests}

The authors declare no competing financial interests. 


\section{Supplementary Files}

This is a list of supplementary files associated with this preprint. Click to download.

- SuppTable1.xlsx

- SuppTable2.xlsx

- SuppTable3.xlsx

- SuppTable4.xlsx

- LNSContogenyepigenomeTFNCSupplement.pdf 